International Journal of Agricultural and Applied Sciences, June 2021, 2(1): 1-23

https://www.agetds.com/ijaas

ISSN: 2582-8053

https://doi.org/10.52804/ijaas2021.211

Review Article

\title{
Review and Updating: Anurans (Amphibia) of Bihar and Jharkhand, India, with some new records from Jehanabad (Bihar) and their role as potential biological controllers
}

\author{
Akhlaq Husain $^{1}$ and Wajid Hasan ${ }^{2}$ \\ ${ }^{1}$ (former Scientist, Zoological Survey of India) \\ 41, Hari Vihar, Vijay Park, Chakrata Road, Dehra Dun - 248001, Uttarakhand, India \\ ${ }^{2}$ Krishi Vigyan Kendra, Jehanabad, BAU, Sabour, India \\ Corresponding author e-mail: drakhlaqhusain@gmail.com
}

(Received: 15/04/2021; Revised: 30/05/2021; Accepted: 24/06/2021)

\begin{abstract}
Present review is meant to update and bring the scattered records of anuran species from Bihar and Jharkhand at one place. A total of 22 valid species (19 from Bihar and 15 from Jharkhand) have been recorded from the existing literature and present new records from Jehanabad (Bihar) are dealt herewith in detail (relevant synonyms, common names, diagnostic features, size, altitudinal records, distribution in Bihar (including new records from Jahanabad), Jharkhand, rest of India and elsewhere and conservation status of each species and their role as potential biological controllers). Variations found in some morphological characters of species studied by various workers from different locations have been recorded.
\end{abstract}

Keywords: Anurans, Bihar and Jharkhand, New Records, Potential Biological Controllers.

\section{INTRODUCTION}

Amphibian fauna of Bihar and Jharkhand has been studied or referred by various workers during the past (Boulenger, 1890, 1920; Annandale and Rao, 1918; Parker, 1934; Venkateswarlu and Murthy, 1972; Bhaduri, 1944; Inger and Dutta, 1986; Ray, 1998; Sarkar, 1991; Chanda, 2002; Sarkar and Ray, 2002; Sarkar et al., 2004; Deuti et al., 2012; Sanyal et al., 2014; Hussain et al., 2017; Kumar, 2019; Prasad et al., 2019; Verma, 2019; Husain and Husain, 2020; Pankaj, 2020; Sharma and Kumar, 2020; Pankaj and Nath, 2021a, b) but no consolidated account is available and hence a detailed updated account of the species with some new records from Jehanabad (Bihar) is provided here. In addition, their use as potential biological controlling agents for insect and other arthropod pests in paddy and other agricultural fields has also been provided. During present study a detailed account of 22 species, belonging to 12 genera, 5 families under order Anura and class Amphibia from Bihar and Jharkhand recorded by earlier workers and present new records (Firouzophrynus stomaticus, Haplobatrachus tigerinus, Uperodon taprobanicus and Polypedates maculatus) from Jehanabad (Bihar) has been provided with their distribution, conservation status and role as potential biological controllers. As regards the diversity, genus Uperodon comprises the maximum number of species, $18.18 \%$, out of total species and its family Microhylidae $36.36 \%$. Fejervarya limnocharis, though it has been reported by various workers (Annandale and Rao, 1918; Boulenger, 1920; Inger and Dutta, 1968 Venkateswarlu and Murthy, 1972; Sanyal et al., 1991; Sarkar, 1991; Sarkar et al., 1992; Ray, 1999; Chanda, 1994, 1995a,b, 2002; Sarkar and Ray, 2002, 2006; Sarkar et al., 2004; Chandra and Gajbe, 2005; Saniyal et al., 2014; Husain, 2015; Kumar, 2019; Pankaj, 2020; Pankaj and Nath, 2021a, b; others) from the area (Bihar and Jharkhand) and rest of Indian mainland (Arunachal Pradesh, Assam, Delhi, Gujarat, Haryana, Himachal Pradesh, Jammu and Kashmir, Kerala, Maharashtra, Manipur, Meghalaya, Mizoram, Nagaland, Punjab, Rajasthan, Sikkim, Tamil Nadu, Tripura, Uttarakhand, Uttar Pradesh and West Bengal) is not included here. It is actually known from Andaman and Nicobar Islands (India), Cambodia, Indonesia, Laos, Malaysia, Myanmar, Thailand and Vietnam as per molecular and morphological studies whereas populations from India (main land), Bangladesh, China, Indonesia, Japan, Nepal, Philippines, Sri Lanka and elsewhere, associated with this name, are currently considered unnamed species as assessed by Frost (2021). Earlier, Sumida et al. (2007) provided evidence that the nominal Fejervarya limnocharis is composed of several species, including unnamed species from India, Japan, Sri Lanka, Taiwan and Thailand. Dinesh et al. (2009) and Dinesh et al. (2020) also didn't include this in Indian fauna. 
Hussain et al. (2017) listed 6 species from Gautam Buddha Wildlife Sanctuary (Bihar and Jharkhand), out of which Bufo bufo and Litoria alboguttata (= Ranoidea alboguttata), the European and Australian forms respectively, appear misidentified for other local species (eg. Duttaphrynus melanostictus and Kaloula assamensis or K. pulchra) and hence not included here. Further, Ranoidea alboguttata belongs to Pelodryadidae, an Australian Tree Frogs family.

\section{BIHAR (Lat. $25^{\circ} 1^{\prime}$ N, Long. $85^{\circ} 32^{\prime} \mathrm{E}$ )}

One of north-eastern states of India, bounded on north by Nepal, east by West Bengal, west by Uttar Pradesh and south Jharkhand, covering an area of $94,163^{2} \mathrm{~km}$, with forests and a number of rivers (Ganga,Sone, Poonpoon, Falgu, Karmanasa, Durgawati, Kosi, Gandak , Ghaghara et al.), reservoirs and ponds and comprising Araria, Arwal, Aurangabad, Banka, Begusarai, Bhagalpur, Bhojpur, Buxar, Darbhanga, East Champaran, Gaya, Gopalganj, Jamui, Jehanabad, Kaimur, Katihar, Khagaria, Kishanganj, Lakhisarai, Madhepura, Madhubani, Munger, Muzaffarpur, Nalanda, Nawada, Patna, Purnea, Rohtas, Saharsa, Samastipur, Saran, Sheikhpura, Sheohar, Sitamarhi, Siwan, Supaul, Vaishali and West Champaran districts. Flora: Common trees include Boswellia serrata (Salai), Diospyros melanoxylon (Kendu), Madhuca indica (Mahua), Pterocarpus marsupium (Paisar), Shorea robusta (Sal), Terminalia arjuna (Arjun), Terminalia bellirica (Bahera), Terminalia tomentosa (Asan), Toona ciliata (Toon) etc.

Climate: Warm and temperate.

\section{JHARKHAND (Lat. $23^{\circ} \mathbf{4 5}^{\prime} \mathrm{N}$, Long. 85 $^{\circ}$ 30 $^{\prime} \mathrm{E}$ )}

It came into existence in 2000, carved out from Bihar. It is bounded by on north by Bihar, east by West Bengal, west by Uttar Pradesh and Chhattisgarh and south by Odisha, covering an area of $79,714^{2} \mathrm{~km}$ with forests $\left(18,324^{2} \mathrm{~km}\right)$, a number of rivers (Ganga, Son, Subarnarekha, Kharkai, Damodar, North Koel, South Koel, Lilajan, Ajay, Mayurakshi and Barakar), reservoirs and ponds and comprising Bokaro, Chatra, Deoghar, Dhanbad, Dumka, East Singhbhum, Garhwa, Giridih, Godda, Gumla, Hazaribagh, Jamtara, , Kharsawan, Khunti, Koderma, Latehar, Lohardaga, Palamu, Pakur, Ramgarh, Ranchi, Sahebganj, Saraikela, Simdega and West Singhbhum districts.

Flora: Acacia spp., Ageratum conyoides, Anogeissus latifolia (Dhaura), Artocarpus heterpphyllus (Kathal), Azadirachta indica (Neem), Bombax ceiba (Semal),Phyllanthus emblica (Aamla), Annona squamosa (Sharifa), Bamboos, Boswellia serrata (Salai), Butea monosperma (Palash), Carica papaya (Papita), Carthemum tinctorius (Kusum), Cleistanthus collinus (Karra), Careya arborea (Wild Guava), cassia spp., Clerodendrum infortunatum (Bhat), Cochlospermum religiosum (Silk Cotton tree), Combretum decandrum (Paibal), Dioscorea spp., Dillenia aurea, Dillenia pentagyna (Karmal), Croton bonplandianus, Eugenia spp., Ficus benghalensis
(Bargad), Ficus religiosa (Peepal), Flacourtia indica (Bilangada), Gardenia spp., Gymnosporia montana (Mountain Spike), Holarrhena spp., Hyptis suaveolens, Indigofera cassioides (Neel), Kydia calycina (Bharanga), Lannea coramandelica (Mohin), Lantana camara, Lygodium spp., Madhuca longifolia (Mahua), Mangifera indica (Mango), Moringa olifera (Sahjan), Phoenix acaulis (Dwarf Date Palm), Phoenix dactylifera (Khajur), Pterocarpus marsupium (Kino), Scoparia dulcis (Goat-weed), Shorea robusta (Sal), Smilax spp., Sterculia urens (Kulu), Tamarindus indica (Imli), Terminalia arjuna (Arjun), and Woodfordia fruticosa (Dhawai).

Climate: Humid subtropical in north to tropical wet and dry in south-east.

SYSTEMATIC ACCOUNT, DISTRIBUTION AND CONSERVATION STATUS

Class: Amphibia Linnaeus, 1758

Order: Anura Dumeril, 1805 (as Anoures)

FAMILY: BUFONIDAE GRAY, 1825

Genus: Duttaphrynus Frost et al., 2006

\section{Duttaphrynus melanostictus (Schneider, 1799)}

Bufo melanostictus Schneider, 1799. Hist. Amph. Nat., 1: 216 (type-locality: India orientali); Annandale and Rao, 1918, Rec. Indian Mus., 15: 38; Inger and Dutta, 1968. J. Bombay nat. Hist. Soc., 83 (suppl.): 136; Vankateswarlu and Murthy, 1972. Indian J. Zool., 13 (3): 129; Sarkar, 1991. Rec. zool. Surv. India, 89 (1-4): 210-211; Chanda, 2002. Handbook Indian Amphibians: 265; Sarkar et al., 2004. Fauna of Bihar (including Jharkhand), Part I, State Fauna Series, 11: 183-184.

Duttaphrynus melanostictus, Sarkar and Ray, 2002. Amphibia. In: Fauna of Kabar Lake. Wetland Ecosystem Series, 4: 107-108; Saniyal et al., 2014. Rec. zool. Surv. India, Occ. Paper No. 361:19; Kumar, 2019. frog leg \# 138. In: Zoo's Print, 34 (8):13, 16, fig.; Pankaj, 2020. International Research Journal of Modernization in Engineering Technology and Science, 2 (1): 78, 79, 80, 81; Pankaj and Nath, 2021a. Reptiles and Amphibians, 28 (1): 107, fig. 2A; Pankaj and Nath, 2021b. MOJ Biology and Medicine, 6 (2): 60-61.

Common Names: Asian Toad, Black-spined Toad, Common Toad, Eyebrow-ridge Toad, Indian Toad.

Diagnostic Features: Head with bony ridges; parotid large, bean or kidney-shaped; Interorbital width broader than upper eyelid; tympanum distinct, circular or oval, closer to eye and $2 / 3^{\text {rd }}-3 / 4^{\text {th }}$ of eye diameter; fingers free, $1^{\text {st }}$ slightly longer than $2^{\text {nd }}$ but shorter than $4^{\text {th }}, 3^{\text {rd }}$ longest, $2^{\text {nd }}$ slightly shorter than $4^{\text {th}}$; tips blunt (Chanda, 1995a) or rounded, not enlarged, without grooves (Dubois and Ohler, 1999) or usually cornified and black (Ray, 1999) or swollen (Sarkar et al., 1992; Sarkar et al.,2004) or without discs (Prasad et al., 2020); subarticular tubercles prominent, all except those of $4^{\text {th }}$ finger double, one prominent and rounded palmer tubercle, numerous small and pointed tubercles on palm and fingers (Dubois and Ohler, 1999); toes nearly half or almost $2 / 3^{\text {rd }}$ webbed, more than three digital phalanges of $4^{\text {th }}$ toe free, $1^{\text {st }}$ toe shortest, $4^{\text {th }}$ longest, $3^{\text {rd }}$ 
longer than $5^{\text {th }}$, tips blunt (Chanda, 1995) or rounded, not enlarged and without grooves (Dubois and Ohler, 1999) or obtuse (Ray, 1999) or swollen (Sarkar et al., 1992; Sarkar et al., 2004); two segments of $4^{\text {th }}$ toe free (Deuti, 2017); subarticular tubercles small and indistinct (Chanda, 1995a) or indistinct with supernumerary tubercles (Dubois and Ohler, 1999); Inner metatarsal tubercle short but very prominent, outer rounded and distinct with numerous tubercles on sole and toes (Dubois and Ohler, 1999) or inner metatarsal tubercle elongated and slightly produced anteriorly, outer oval (Ray, 1999); both inner and outer metatarsal tubercles equal in size (Deuti et al., 2017); inner metatarsal tubercle small and distinct (Prasad et al., 2020); tibio-tarsal articulation reaching shoulder or

Table 1. Showing distribution of species in Bihar and Jharkhand with their Conservation Status (IUCN Red List= International Union for Conservation of Nature, $\mathrm{LC}=$ Least Concern).

\begin{tabular}{|c|c|c|c|c|c|c|}
\hline $\begin{array}{l}\text { Sl. } \\
\text { No. }\end{array}$ & Family & Genus & Species & Bihar & Jharkhand & $\begin{array}{l}\text { IUCN Rec } \\
\text { List Status }\end{array}$ \\
\hline 1. & $\begin{array}{l}\text { Bufonidae Gray, } \\
1825\end{array}$ & $\begin{array}{l}\text { Duttaphrynus Frost et } \\
\text { al., } 2006\end{array}$ & $\begin{array}{l}\text { Duttaphrynus melanostictus } \\
\text { (Schneider, 1799) }\end{array}$ & + & + & $\mathrm{LC}$ \\
\hline 2. & -do- & $\begin{array}{l}\text { Firouzophrynus } \\
\text { Safaei-Mahroo and } \\
\text { Ghaffari, } 2020\end{array}$ & $\begin{array}{l}\text { Firouzophrynus stomaticus } \\
\text { Luetkin, } 1864\end{array}$ & + & + & $\mathrm{LC}$ \\
\hline 3. & $\begin{array}{l}\text { Dicroglossidae } \\
\text { Anderson, } 1871\end{array}$ & $\begin{array}{l}\text { Euphlyctis } \\
\text { Fitzinger, } 1843\end{array}$ & $\begin{array}{l}\text { Euphlyctis cyanophlyctis } \\
\text { (Schneider, 1799) }\end{array}$ & + & + & $\mathrm{LC}$ \\
\hline 4. & - do- & $\begin{array}{l}\text { Hoplobatrachus } \\
\text { Peters, } 1863\end{array}$ & $\begin{array}{l}\text { Hoplobatrachus } \quad \text { crassus } \\
\text { (Jerdon, 1853) }\end{array}$ & + & + & $\mathrm{LC}$ \\
\hline 5. & - do- & -do- & $\begin{array}{l}\text { Hoplobatrachus } \quad \text { tigerinus } \\
\text { (Daudin, 1802) }\end{array}$ & $+\varnothing$ & + & $\mathrm{LC}$ \\
\hline 6. & - do- & $\begin{array}{l}\text { Sphaerotheca } \\
\text { Guenther, } 1859\end{array}$ & $\begin{array}{ll}\text { Sphaerotheca } & \text { breviceps } \\
\text { (Schneider, 1799) } & \end{array}$ & + & + & $\mathrm{LC}$ \\
\hline 7. & - do- & -do- & $\begin{array}{l}\text { Sphaerotheca magadha (Prasad } \\
\text { et al., } 2019\end{array}$ & -7 & + & $\mathrm{LC}$ \\
\hline 8. & - do- & -do- & $\begin{array}{l}\text { Sphaerotheca rolandae (Dubois, } \\
\text { 1983) }\end{array}$ & + & - & $\mathrm{LC}$ \\
\hline 9. & - do- & $\begin{array}{l}\text { Occidozyga Kuhl and } \\
\text { Van Hasselt, } 1822\end{array}$ & $\begin{array}{l}\text { Occidozyga lima (Gravenhorst, } \\
1829 \text { ) }\end{array}$ & + & + & $\mathrm{LC}$ \\
\hline 10. & $\begin{array}{l}\text { Microhylidae } \\
\text { Guenther, } 1858\end{array}$ & Kaloula Gray, 1831 & $\begin{array}{l}\text { Kaloula assamensis Das et al., } \\
2005\end{array}$ & + & - & $\mathrm{LC}$ \\
\hline 11. & - do- & -do- & Kaloula pulchra Gray, 1831 & + & - & $\mathrm{LC}$ \\
\hline 12. & - do- & $\begin{array}{l}\text { Microhyla Tschudi, } \\
1838\end{array}$ & $\begin{array}{l}\text { Microhyla ornata (Dumeril and } \\
\text { Bibron, 1841) }\end{array}$ & + & + & $\mathrm{LC}$ \\
\hline 13. & - do- & - do- & Microhyla rubra (Jerdon, 1854) & + & - & $\mathrm{LC}$ \\
\hline 14. & - do- & $\begin{array}{l}\text { Uperodon Dumeril and } \\
\text { Bibron, } 1841\end{array}$ & $\begin{array}{ll}\text { Uperodon } & \text { globulosus } \\
\text { (Guenther, 1864) } & \end{array}$ & + & + & $\mathrm{LC}$ \\
\hline 15. & - do- & -do- & $\begin{array}{l}\text { Uperodon systoma (Schneider, } \\
1799 \text { ) }\end{array}$ & + & + & $\mathrm{LC}$ \\
\hline 16. & $-\mathrm{d} 0-$ & -do- & $\begin{array}{ll}\text { Uperodon } & \text { taprobanicus } \\
\text { (Parker, 1934) } & \end{array}$ & + & + & $\mathrm{LC}$ \\
\hline 17. & - do- & -do- & $\begin{array}{ll}\text { Uperodon } & \text { variegatus } \\
\text { (Stoliczka, 1872) } & \end{array}$ & - & + & $\mathrm{LC}$ \\
\hline 18. & $\begin{array}{l}\text { Ranidae Batsch, } \\
1796\end{array}$ & $\begin{array}{l}\text { Hylarana Tschudi, } \\
1838\end{array}$ & $\begin{array}{l}\text { Hylarana erythraea (Schlegel, } \\
\text { 1837) }\end{array}$ & + & - & $\mathrm{LC}$ \\
\hline 19. & $\begin{array}{l}\text { Rhacophoridae } \\
\text { Hoffman, } 1932\end{array}$ & $\begin{array}{l}\text { Chirixalus } \\
\text { Boulenger, } 1893\end{array}$ & $\begin{array}{l}\text { Chirixalus simus Annandale, } \\
1915\end{array}$ & - & + & $\mathrm{LC}$ \\
\hline 20. & - do- & $\begin{array}{l}\text { Polypedates } \\
\text { Tschudi, } 1838\end{array}$ & $\begin{array}{l}\text { Polypedates leucomystax } \\
\text { (Gravenhorst, 1829) }\end{array}$ & + & - & $\mathrm{LC}$ \\
\hline 21. & - do- & -do- & $\begin{array}{l}\text { Polypedates maculatus (Gray, } \\
\text { 1830) }\end{array}$ & + & + & $\mathrm{LC}$ \\
\hline 22. & - do- & -do- & $\begin{array}{l}\text { Polypedates } \\
\text { (Boulenger, 1906) }\end{array}$ & + & - & $\mathrm{LC}$ \\
\hline Total & 5 & 12 & 22 & 19 & 15 & 22 \\
\hline
\end{tabular}


12-116 mm (Sarkar et al., 2004); $150 \mathrm{~mm}$ (Ingle, 2011); 48-76 and 45-109 mm (Deuti et al., 2014); $165 \mathrm{~mm}$ (Husain, 2015); 48.34-82.76 and 45-115 mm (Deuti et al., 2017); 45-95 mm (ingle, 2020); male $58.12 \mathrm{~mm}$ (Prasad et al., 2020); about $20 \mathrm{~cm}$ (wikipedia).

Altitudinal Records: Up to $3,048 \mathrm{~m} / 10,000 \mathrm{ft}$ in Sikkim Himalaya (Boulenger, 1890); 1,356 m / 4,450 $\mathrm{ft}$, Bhimtal and Teesta river (Annandale and Rao, 1918); throughout plains (Sarkar, 1991); about 1,400 m in Darjeeling Himalaya (Sarkar et al., 1992); up to 3,048 m (Chanda, 1995a,b); 2,500-3,000 m (Ray, 1999); $815 \mathrm{~m}$ at Araku, Andhra Pradesh; $875 \mathrm{~m}$ at Koraput,370 m at Rayagada and $435 \mathrm{~m}$ at Semiliguda, Odisha(Deuti et al., 2014); up to 2,500 m (Husain, 2015); 2,000 m (Frost, 2021).

\section{Distribution:}

Bihar: Aurangabad (Raja Jagganath Dam Deo), Begusarai (Kabar Lake), East Champaran, Darbhanga, Gaya (Gautam Buddha Wildlife Sanctuary), Nalanda, Saran (Siripur) and West Champaran (Valmiki Tiger Reserve) districts.

Jharkhand: Deoghar (Sarsa), Dhanbad, Dumka, East Singhbhum (Jamshedpur and Chakulia), Gumla, Hazaribagh, Koderma (Gautam Buddha Wildlife Sanctuary), Ranchi, Santhal Pargana and West Singhbhum districts.

Rest of India: Andhra Pradesh (Araku), Arunachal Pradesh, Assam, Chandigarh, Chhattisgarh (Bastar, Bijapur, Dhamtari, Dantewada, Jashpur, Kanker, Kondagaon, Narainpur and Sukma dists.; Indravati Tiger Reserve, Bijapur dist.; Kanger Valley National Park, Bastar dist.), Delhi, Gujarat (Bhavnagar, Dang, Junagarh and Sabar Kantha dists.), Haryana, Himachal Pradesh (Mashobra, Shimla Hills), Jammu and Kashmir, Karnataka (Bengaluru Urban, Hassan, Haveri, Kodagu, Shimoga and Shivamogga dists.), Kerala (Idukki, Kollam, Kottayam, Pathanamthitta and Thrissur dists.), Lakshadweep (Lakshadweep dist.), Madhya Pradesh (Balaghat, Bhopal, Dinori, Gwalior, Jabalpur, Mandla Morena, Seoni, Shivpuri and Ujjain dists.; Kanha Tiger Reserve, Balaghat and Mandla dists.; Panna Tiger Reserve, Panna and Chhatarpur dists.; Pench Tiger Reserve, Seoni dist.), Maharashtra (Ahmadnagar, Mumbai Suburban, Pune, Raigad, Ratnagiri, Sangli, Satara, Sindhudurg and Thane dists.), Manipur, Meghalaya (Khasi Hills; Garo Hills), Mizoram, Nagaland, Odisha (Bhubaneshwar, Koraput and Rayagada dists.), Rajasthan (Ajmer and Jhalwar dists.; Aravalli), Sikkim, Tamil Nadu (Chennai, Injambakkam, Krishnagiri, Nilgiris and Tirunelveli dists.), Telangana (Hyderabad, Mahbubnagar, Medak and Rangareddy dists.), Tripura (West Tripura dist.), Uttarakhand (Almora, Chamoli, Dehra Dun, Haridwar, Nainital, Pauri, Pithoragarh, Tehri and Uttarkashi dists.; Corbett Tiger Reserve, Nainital and Pauri dists.; Govind Pashu Vihar, Uttarkashi dist.; Nanda Devi Biosphere Reserve, Chamoli dist.; Rajaji Tiger Reserve, Dehra Dun, Haridwar and Pauri dists.), Uttar Pradesh (Azamgarh, Lucknow and Prayagraj dists.) and West
Bengal (Bankura, Bardhaman, Birbhum, Cooch Behar, Darjeeling, Jalpaiguri- Chilapata Forest, Gorumara National Park, Jaldapara National Park and Mahananda Wildlife Sanctuary; Kolkata, Malda, Midnapore, Murshidabad, Nadia, 24 Parganas, Purulia, West Dinajpur dists.; Buxa Tiger Reserve, Alipurduar dist.). Introduced: Andaman and Nicobar Islands.

Elsewhere: Bangladesh, Brunei, Cambodia, China, Hong Kong, Indonesia, Laos, Malaysia, Maldives, Myanmar, Nepal, Pakistan, Philippines, Singapore, Sri Lanka, Taiwan, Thailand and Viet Nam.

Introduced: Sulawesi, East Timor, Papua New Guinea and Madagascar (Frost, 2021).

Uncertain: Bhutan (iucnredlist).

Conservation Status: IUCN Red List- Least Concern.

Remarks: Hussain et al. (2017) recorded it as Bufo bufo (Linnaeus, 1758) from Gautam Buddha Wildlife Sanctuary (Bihar and Jharkhand) which appears a misidentification, as it is found in Balkans, France, Italy, Russian, Turkey and Ukraine. It might have been identified for Duttaphrynus melanostictus, the Common Toad.

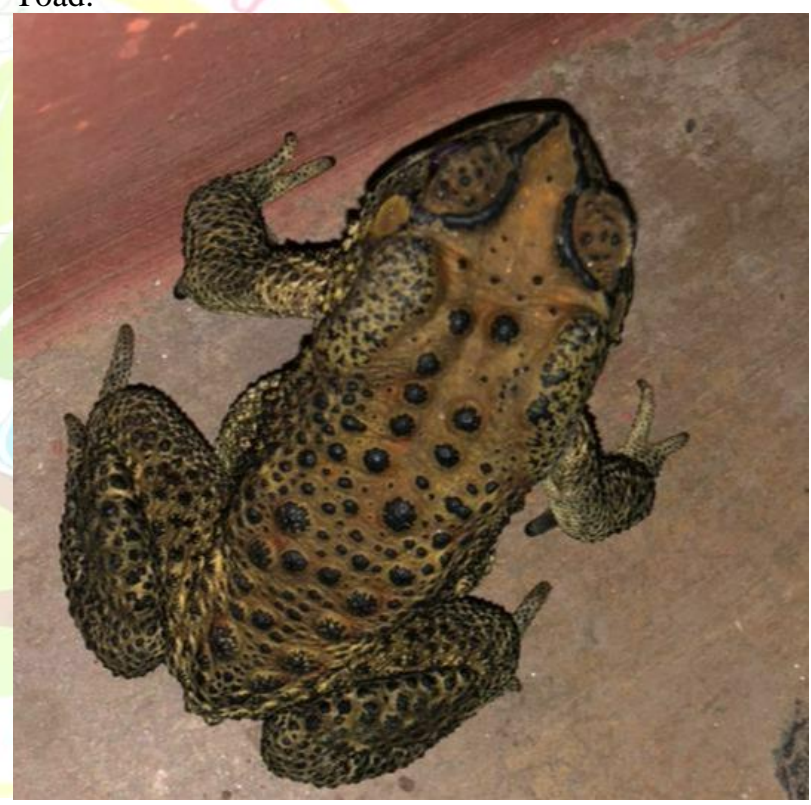

Figure 1. Duttaphrynus melanostictus, Common Toad (Credit: Dr. H. J. Husain)

Genus: Firouzophrynus Safaei-Mahroo and Ghaffari, 2020.

2. Firouzophrynus stomaticus (Luetken, 1864)

Bufo stomaticus Luetken, 1864 (1863). Vidensk. Medd. Dansk. Naturhist. Foren., Ser. 2, 4: 305 (type-locality: ostindiske [East Indies]); Inger and Dutta, 1968. J. Bombay nat. Hist. Soc., 83 (suppl.): 136; Vankateswarlu and Murthy, 1972. Indian J. Zool., 13 (3): 130; Sarkar, 1991. Rec. zool. Surv. India, 89 (1-4): 210-211; Ray, 1999. Mem. zool. Surv. India, 18 (3): 6467, pl. 22, figs.1-4; Chanda, 2002. Hand book Indian amphibians: 28, 265; Sarkar et al., 2004. Fauna of Bihar (including Jharkhand), Part I, State Fauna Series, 11: 184. 
Duttaphrynus stomaticus, Saniyal et al., 2014. Rec. zool. Surv. India, Occ. Paper No. 361:19; Kumar, 2019. frog leg \# 138. In: Zoo's Print, 34 (8):13, 15, fig.; Pankaj, 2020. International Research Journal of Modernization in Engineering Technology and Science, 2 (1): 78, 79, 80, 81; Pankaj and Nath, 2021a. Reptiles and Amphibians, 28 (1): 107, fig. 2B; Pankaj and Nath, 2021b. MOJ Biology and Medicine, 6 (2): 60-61.

Firouzophrynus stomaticus, Safaei-Mahroo and Ghaffari. 2020. The Complete Guide to Amphibians of Iran: Biology, Ecology, and Conservation. Sanandaj: 20. University of Kurdistan Press, Iran.

Common Names: Assam Toad, Marbled Toad, IndoGangetic Toad, Indus Toad.

Diagnostic Features: Head without bony ridges; parotid large, elliptical and flattened (not kidneyshaped); interorbital width broader than upper eyelid; tympanum distinct, circular or oval, slightly more than $1 / 2,2 / 3^{\text {rd }}$ or nearly one eye diameter; fingers free, $1^{\text {st }}$ little longer than $2^{\text {nd }}, 3^{\text {rd }}$ longest, $4^{\text {th }}$ shortest; tips of fingers and toes swollen (Sarkar et al., 1992; Sarkar et al., 2004); subarticular tubercles blunt, mixed with flat palmer glands; palmer tubercle distinct, triangular and wider in front; toes more than $1 / 2^{\text {nd }}$ to $2 / 3^{\text {rd }}$ webbed, two phalanges of $4^{\text {th }}$ toe free; toes $2 / 3^{\text {rd }}$ webbed (Ray, 1999; Chanda, 2002) or scarcely webbed (Prasad et al., 2020); subarticular tubercles small; inner and outer metatarsal tubercles sharp-edged and equal sized (Chanda, 2002); tibio-tarsal articulation reaching between tympanum and eye (Sarkar et al., 1992; Sarkar et al., 2004) or hind edge of eye (Ray, 1999; Husain et al., 2020) or between shoulder and eye (Chanda, 2002). Colouration: Brownish, greyish or olive-brown with smooth warts and mixed grey blotches; fingertips black; vocal sac of calling male white or yellowish.

Snout-Vent Length: $76.2 \mathrm{~mm} / 3$ in (Boulenger, 1890); 27-79 mm (Sarkar, 1991); 8-86 mm (Sarkar et al., 1992); 10-80 mm (Ray and Tilak, 1995); juvenile 10-30 $\mathrm{mm}$, adult $76 \mathrm{~mm}$ (Chanda, 2002); 12-75 mm (Sarkar et al, 2004); $90 \mathrm{~mm}$ (Ingle, 2011); 52-67 and 38-76 mm (Deuti et al., 2014); $90 \mathrm{~mm}$ (Husain, 2015); 42-86 mm (Deuti et al., 2017); male $47.5 \mathrm{~mm}$ (Prasad et al., 2020).

Altitudinal Records: $1829 \mathrm{~m} / 6,000 \mathrm{ft}$ in Nepal (Annandale and Rao, 1918); 100-1,828 $\mathrm{m}$ in Nepal (Chanda, 2002); $230 \mathrm{~m}$ at Kesinga and $435 \mathrm{~m}$ at Semiliguda, Odisha (Deuti et al., 2014); up to $4,500 \mathrm{~m}$ (Husain, 2015; iucnredlist).

\section{Distribution:}

New Record: Gandhar and Safepur, Jehanabd (Bihar).

Bihar: Aurangabad (Raja Jagannath Dam Deo), Bhojpur, East Champaran, Gaya, Nalanda, Palamau, Patna, Sahibganj, Saran and Vaishali districts.

Jharkhand: Chota Nagpur, East Singhbhum and Ranchi districts.

Rest of India: Andhra Pradesh, Assam, Chandigarh, Chhattisgarh (Jashpur dist.), Delhi, Gujarat (Ahmedabad, Bhavnagar, Patan and Vadodara dists.), Haryana, Himachal Pradesh, Jammu and Kashmir, Karnataka (Tumakuru and Coorg/ Kodagu dists.),
Kerala, Madhya Pradesh (Gwalior and Morena dists.; Panna Tiger Reserve, Panna and Chhatarpur dists.), Maharashtra (Ahmadnagar, Chandrapur, Jalgaon, Pune, Sangli, Satara and Solapur dists.), Manipur, Odisha (Kalahandi and Koraput dists.), Punjab (Bathinda and Moga dists.), Rajasthan (Ajmer, Jaisalmer, Jhalawar and Udaipur dists.), Sikkim, Tamil Nadu (Chennai dist.), Uttarakhand (Almora, Dehra Dun, Nainital, Pauri, Pithoragarh, Tehri and Udham Singh Nagar dists.; Corbett Tiger Reserve, Nainital and Pauri dists.; Rajaji Tiger Reserve, Dehra Dun, Haridwar and Pauri dists.), Uttar Pradesh (Agra, Aligarh, Gautam Buddh Nagar, Lucknow and Mirzapur dists.; Katarniaghat Wildlife Sanctuary, Bahraich dist.) and West Bengal (Bankura, Bardhaman, Birbhum, Cooch Behar, Jalpaiguri, Malda, Midnapore, Nadia, Purulia and 24 Parganas dists.) and Western Ghats.

Elsewhere: Afghanistan, Bangladesh, China, Iran, Myanmar, Nepal, Oman and Pakistan.

Introduced: Sri Lanka (Manamendra-Arachchi and Pethiyagoda, 1998).

Uncertain: Bhutan and China (iucnredlist).

Records from Arabia (Ray and Tilak, 1995; Sarkar et al., 2004) need confirmation.

Conservation Status: IUCN Red List- Least Concern.

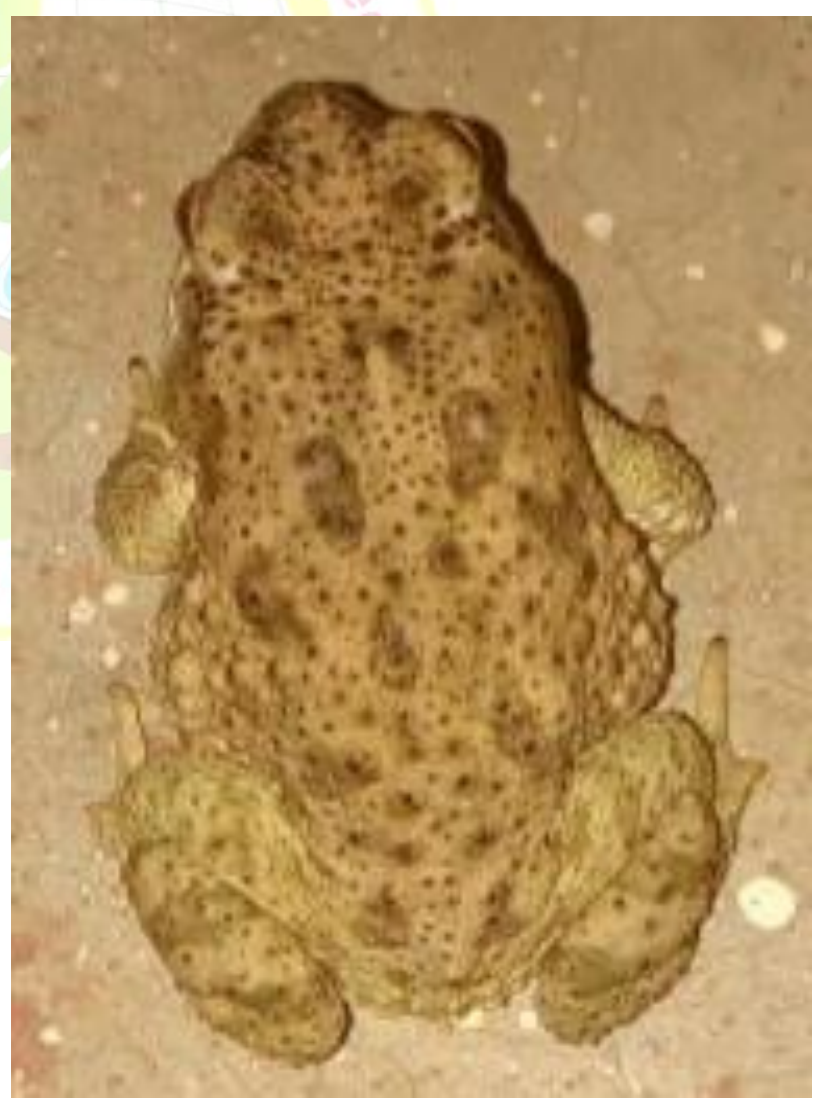

Figure 2a. Firouzophrynus stomaticus, Marbled Toad (without verterbral stripe, from Jehanabad, Bihar; Credit: Kumar Sahab) 


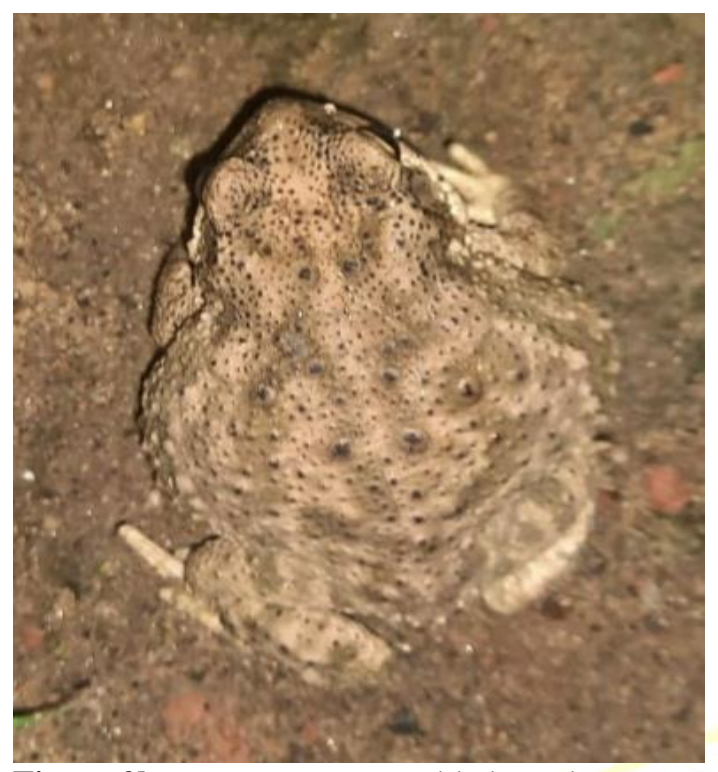

Figure 2b. F. stomaticus, Marbled Toad (with faint vertebral stripe, from Jehanabd, Bihar; Credit: Mr. Ramlakhan Thakur)

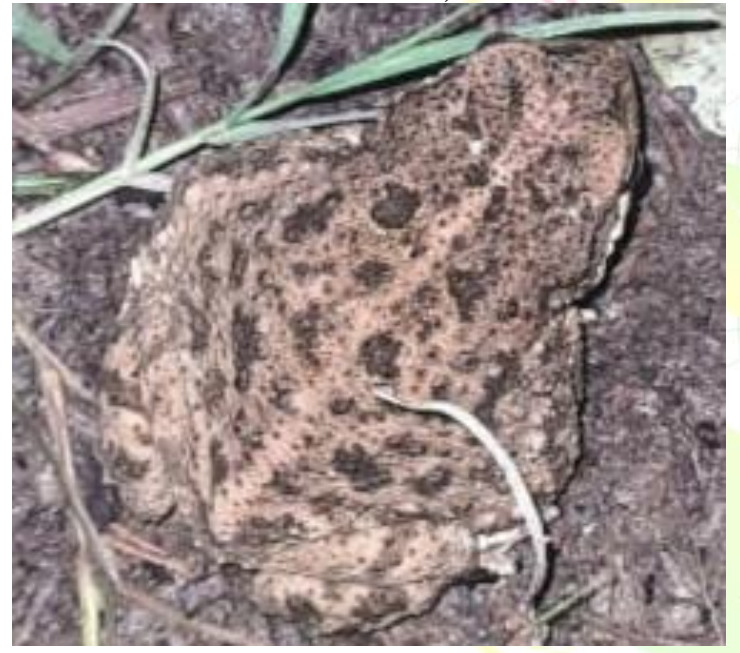

Figure 2c. $F$. stomaticus (with distinct vertebral stripe, from Jehanabd, Bihar; Credit: Mr. Ramlakhan Thakur)

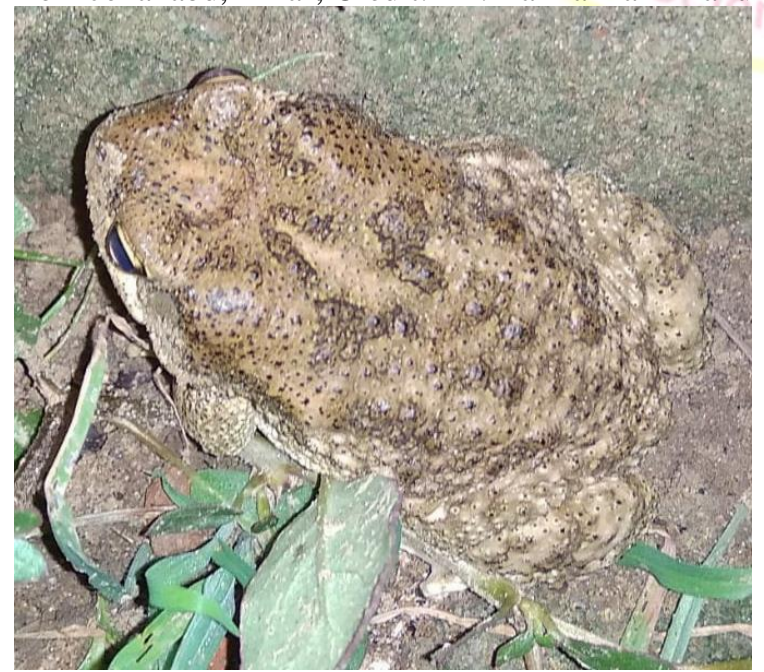

Figure 2d. F. stomaticus (in bushes for feeding, from Jehanabad, Bihar; Credit: Kumar Sahab)

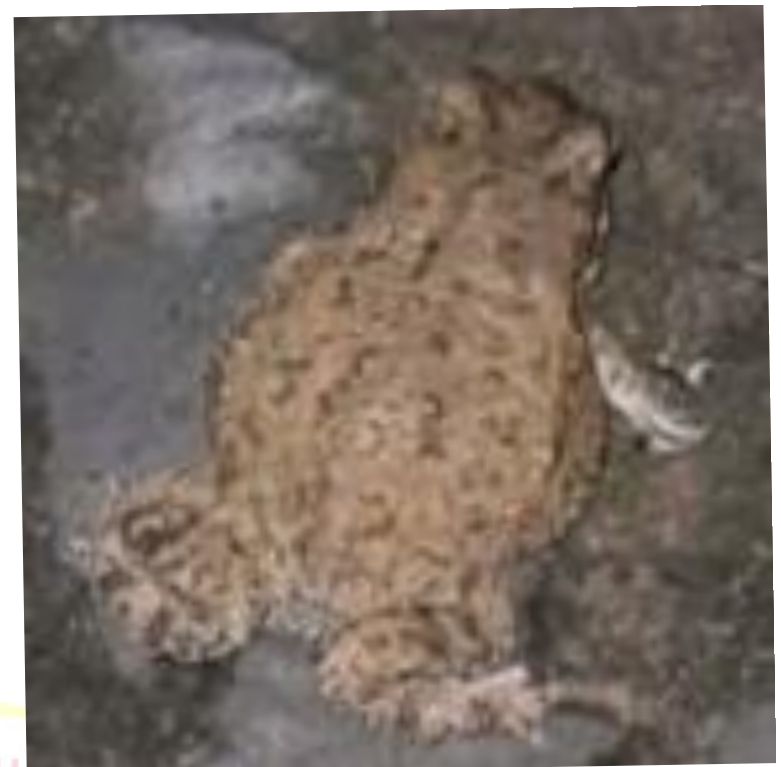

Figure 2e. F. stomaticus (juvenile, from Jehanabad, Bihar; Credit: Mr. Ramlakhan Thakur)

FAMILY: DICROGLOSSIDAE ANDERSON, 1871 Subfamily: Dicroglossinae Anderson, 1871

Genus: Euphlyctis Fitzinger, 1843

3. Euphlyctis cyanophlyctis (Schneider, 1799)

Rana cyanophlyetis Schneider, 1799. Hist. Amph., 1: 137, 144 (type-locality: India orientali) (cyanophlyctis as per Code of Zoological Nomenclature (1999), Art. 33.3.1); Annandale and Rao, 1918. Rec. Indian Mus., 15 (1): 30-31; Venkateswarlu and Murthy, 1972. Indian J. Zool, 13 (3): 129; Sarkar, 1991. Rec. zool. Surv. India, 89 (1-4): 213-214; Chanda, 2002. Handbook Indian Amphibians: 265; Sarkar and Ray, 2002. Amphibia. In: Fauna of Kabar Lake. Wetland Ecosystem Series, 4: 109-110; Sarkar et al., 2004. Fauna of Bihar (including Jharkhand), Part I, State Fauna Series, 11: 188.

Euphlyctis cyanophlyctis, Rao and Shukla, 2014. International Research Journal of Natural and Applied Sciences, 1 (6): 186-190, figs. 1a, b; Saniyal et al., 2014. Rec. zool. Surv. India, Occ. Paper No. 361:20; Hussain et al., 2017. An assessment report on biodiversity value of Gautam Buddha Wildlife Sanctuary, Bihar and Jharkhand: 22; Kumar, 2019. frog leg \# 138. In: Zoo's Print, 34 (8):13, 14, fig.; Pankaj, 2020. International Research Journal of Modernization in Engineering Technology and Science, 2 (1): 79, 80, 81; Pankaj and Nath, 2021a. Reptiles and Amphibians, 28 (1): 107, fig. 2C; Pankaj and Nath, 2021b. MOJ Biology and Medicine, 6 (2): 61.

Common Names: Skittering Frog, Skipping Frog, Studded Frog, Water Skipper.

Diagnostic Features: Interorbital width much smaller than upper eyelid or equal to internarial space; tympanum distinct, more than $1 / 2,4 / 5^{\text {th }}$ or nearly one eye diameter; fingers free, $1^{\text {st }}$ equal or almost equal to $2^{\text {nd }}$, $3^{\text {rd }}$ longest, $4^{\text {th }}$ almost equal to $2^{\text {nd }}$, tips obtusely 
pointed; subarticular tubercles of fingers and toes small, feebly prominent (Chanda, 1995a; Sarkar and Ray, 2006) or swollen (Sarkar and Ray, 2002); toes fully webbed, tips swollen (Chanda, 1995a; Sarkar and Ray, 2006) or dilated into small discs (Chanda, 2002), $4^{\text {th }}$ toe longest; inner metatarsal tubercle small, distinct, pointed and digitiform, outer metatarsal tubercle absent; tibio-tarsal articulation reaching between hind end of tympanum and nostril (Sarkar and Ray, 2002, 2006 ) or between eye and nostril (Chanda, 1995a, 2002); hind limbs when folded at right angles to body, heels not touching each other.

Colouration: Brownish, greyish or olive-brownish, spotted or marbled with black or dark olive roundish markings and small warts; vocal sacs of calling male blackish.

Snout-Vent Length: About 65-75 mm (Annandale and Rao, 1918); 38-92 mm (Boulenger, 1920); 22-65 mm (Sanyal et al., 1991); 26-71 mm (Sarkar, 1991); 44.5$66.5 \mathrm{~mm}$ (Chanda, 1994); 40-55 mm (Chanda, 1995b); 21-53 mm (Ray and Tilak, 1995); $65 \mathrm{~mm}$ (Chanda, 2002); 17-73 mm (Sarkar et al., 2004); 17-69 (Sarkar and Ray, 2006); 33-52 and 30-70 mm (Deuti et al., 2014); 114-152 mm / 4.5-6.0 in (Rao and Shukla, 2014); 64 mm (Husain, 2015); 30-70 mm (Deuti et al., 2017); 30-60 mm (Ingle, 2020); males 38.1-44.7, females 55.2-59.5 mm (Prasad et al., 2020).

Altitudinal Records: $732 \mathrm{~m} / 2,400 \mathrm{ft}$ at Kalka and ascends Himalayas at least $1,829 \mathrm{~m} / 6,000 \mathrm{ft}$ (Annandale and Rao, 1918); up to $1,800 \mathrm{~m}$, India (Ray and Tilak, 1995); up to 2,000 m (Ray, 1999); up to $1846 \mathrm{~m}$ in Himalayas (Sarkar, 1991; Sarkar and Ray, 2002); $129 \mathrm{~m}$ at Renigunta, Andhra Pradesh; $230 \mathrm{~m}$ at Kesinga, $355 \mathrm{~m}$ at Muniguda, $370 \mathrm{~m}$ at Rayagada and $435 \mathrm{~m}$ at Semiliguda, Odisha (Deuti et al., 2014); 2,500 m (iucnredlist).

\section{Distribution:}

Bihar: Aurangabad (Raja Jagganath Dam Deo), Bhagalpur, Bhojpur, Chhapra, East Champaran, Garhwa, Gaya (Gautam Buddha Wildlife Sanctuary), Jehanabad, Khagaria, Kaimur (Bhabua), Muzaffarpur, Nalanda, West Champaran, Patna, Saharsa and Saran (Chupra) districts.

Jharkhand: Bokaro (Chas), Dhanbad, East Singhbhum, Hazaribagh, Godda, Gumla, Koderma (Gautam Buddha Wildlife Sanctuary), Palamu, Ranchi, Sahibganj, Santhal Pargana and West Singhbhum districts.

Rest of India: Andhra Pradesh (Chittoor dist.), Arunachal Pradesh (East and West Kameng, East and West Siang, Lower Subansiri and Tirap dists.), Assam, Chhattisgarh (Bastar, Bijapur, Bilaspur, Dhamtari, Dantewada, Jashpur, Kanker, Kondagaon, Narainpur and Sukma dists.; Indravati Tiger Reserve, Bijapur dist.; Kanger Valley National Park, Bastar dist.), Delhi, Goa (North Goa and South Goa dists.), Gujarat (Dang, Gandhinagar and Junagadh dists.), Haryana (Panchkula dist.), Himachal Pradesh, Jammu and Kashmir, Karnataka (Bengaluru Urban, Shivamogga, Udupi and Uttara Kannada dists.), Kerala (Idukki, Kannur,
Kollam, Thrissur and Thiruvananthapuram dists.), Madhya Pradesh (Balaghat, Gwalior, Jabalpur, Mandla, Morena Shivpuri and Ujjain dists.; Kanha Tiger Reserve, Balaghat and Mandla dists.; Panna Tiger Reserve, Panna and Chhatarpur dists.), Maharashtra (Palghar, Pune, Ratnagiri, Sangli, Satara, Sindhudurg and Thane dists.), Manipur, Meghalaya, Mizoram, Nagaland, Odisha (Kalahandi, Koraput, Puri and Rayagada dists.; Barkuda Island, Chilka Lake, Ganjam dist.), Punjab, Rajasthan (Ajmer, Jhalawar and Pali dists.; Aravalli Range), Tamil Nadu, Telangana (Mahabubnagar dist.), Tripura, Uttarakhand (Almora, Dehra Dun, Nainital, Pauri, Pithoragarh, Tehri and Uttarkashi dists.; Gobind Pashu Vihar, Uttarkashi dist.; Rajaji Tiger Reserve, Dehra Dun, Haridwar and Pauri dists.), Uttar Pradesh (Hamirpur and Mirzapur dists.), West Bengal (Bardhaman, Jalpaiguri, Kolkata, Midnapur and South 24 Parganas dist.; Buxa Tiger Reserve, Jaldapara National Park and Kodalbasti Forest, Alipurduar dist.; Gorumara National Park and Mahananda Wildlife Sanctuary, Jalpaiguri dist.) and Western Ghats.

Doubtful: Sikkim (Subba et al., 2016).

Elsewhere: Afghanistan, Bangladesh, Bhutan, Iran, Malaysia, Myanmar, Nepal, Pakistan, Sri Lanka and Viet Nam.

Introduced (Taylor, 1962) / in error (iucnredlist): Thailand.

Records from Saudi Arabia ((Ray and Tilak, 1995; Sen and Mathew, 2008; others) need confirmation.

Frost (2021) reported its distribution from south-eastern Iran, southern Afghanistan, Pakistan, low elevation Nepal, southern Bhutan and India at low to moderate elevations south to Madhya Pradesh and east through Bangladesh and north-eastern India to extreme western Myanmar; Sri Lanka; Malaya; Vietnam.

Conservation Status: IUCN Red List- Least Concern.

Remarks: Rao and Shukla (2014) noted cannibalism in this species.

\section{Genus: Hoplobatrachus Peters, 1863}

\section{Hoplobatrachus crassus (Jerdon, 1863)}

Rana crassa Jerdon, 1863. Asiat. Soc. Bengal, 22: 531 (type-locality: found in a few tanks in the Carnatic [now part of Tamil Nadu and Andhra Pradesh, India]); Annandale and Rao, 1918. Rec. Indian Mus., 15 (3): 2540; Bhaduri, 1947. J. Bombay nat. Hist. Soc., 44 (3-4): 481-484; Venkateswarlu and Murthy, 1972. Indian J. Zool, 13 (3): 129; Inger and Dutta, 1986. J. Bombay nat. Hist. Soc., 83 (suppl.): 137; Sarkar, 1991. Rec. zool. Surv. India, 89 (1-4): 213, 215; Chanda, 2002. Handbook Indian Amphibians: 97, 265; Sarkar et al., 2004. Fauna of Bihar (including Jharkhand), Part I, State Fauna Series, 11: 189-190.

Hoplobatrachus crassa, Saniyal et al., 2014. Rec. zool. Surv. India, Occ. Paper No. 361:20.

Hoplobatrachus crassus, Kumar, 2019. frog leg \# 138. In: Zoo's Print, 34 (8):13, 14-15, fig.; Pankaj, 2020. International Research Journal of Modernization in 
Engineering Technology and Science, 2 (1): 79, 80, 81; Pankaj and Nath, 2021a. Reptiles and Amphibians, 28 (1): 107, fig. 2E; Pankaj and Nath, 2021b. MOJ Biology and Medicine, 6 (2): 60-61.

Common Names: Jerdon's Bull-frog, South Indian Bull-frog.

Diagnostic Features: Interorbital width much smaller than upper eyelid; tympanum distinct, $2 / 3^{\text {rd }}, 3 / 4^{\text {th }}$ or nearly equal of eye diameter; fingers free, tips obtusely pointed (Ray, 1999), $1^{\text {st }}$ longer than $2^{\text {nd }}, 3^{\text {rd }}$ longest, $4^{\text {th }}$ little longer than $2^{\text {nd }}$; subarticular tubercles of fingers and toes small, feebly distinct; toes fully webbed, penultimate phalange of $4^{\text {th }}$ toe free; tips not pointed (Sarkar et al., 1992) or swollen (Ray, 1999; Chanda, 2002) or slightly rounded (Prasad et al., 2020); inner metatarsal tubercle well developed, shovel-shaped and sharp-edged and outer metatarsal tubercle absent (Chanda, 2002; Prasad et al., 2020; Deuti et al., 2017); tibio-tarsal articulation reaching tympanum or eye (Sarkar et al., 1992; Chanda, 2002) or hind margin of tympanum but not beyond (Ray, 1999); hind limbs when folded at right angle to body, heals not overlap.

Colouration: Greyish, greyish-brown, greenish or olivegreen with irregular dark blotches, interrupted long warts and often a light vertebral line.

Snout-Vent Length: 76-112 mm (Boulenger, 1920); males 74.0-77.5, females 65-86 mm (Bhaduri, 1944); 57-73 mm (Sarkar, 1991); 20-100 mm (Sarkar et al., 1992); 47-72 mm (Ray and Tilak, 1995); $120 \mathrm{~mm}$ (Chanda, 2002); 36-72 mm (Sarkar et al., 2004); 57-94 and 40-86 mm (Deuti et al., 2014);130 mm (Husain, 2015); 40-86 mm (Deuti et al., 2017); 80-100 mm (Ingle, 2020); male 70.2, individual (sex not known) $92.2 \mathrm{~mm}$ (Prasad et al., 2020).

Altitudinal Records: $500 \mathrm{~m}$ at Rajaji Tiger Reserve, Uttarakhand (Ray and Tilak, 1995); $129 \mathrm{~m}$ at Renigunta, Andhra Pradesh; $230 \mathrm{~m}$ at Kesinga and 370 $\mathrm{m}$ at Rayagada, Odisha (Deuti et al., 2014); up to $600 \mathrm{~m}$ (Husain, 2015; iucnredlist), below $400 \mathrm{~m}$, Nepal (Frost, 2021).

\section{Distribution:}

Bihar: Aurangabad (Raja Jagannath Dam Deo), Bhagalpur, Gaya, Kaimur (Bhabua), Nalanda, Siwan (Hasua village) and Vaishali districts.

Jharkhand: Dhanbad and Ranchi districts.

Rest of India: Andhra Pradesh (Chittoor dist.), Arunachal Pradesh, Assam, Chhattisgarh (Bastar, Bijapur, Bilaspur, Dantewada, Kanker, Kondagaon, Narainpur and Sukma dists.; Indravati Tiger Reserve, Bijapur dist.; Kanger Valley National Park, Bastar dist.), Karnataka, Kerala (Malabar dist.), Madhya Pradesh (Panna Tiger Reserve, Panna and Chhatarpur dists.), Maharashtra, Nagaland, Odisha (Bhadrak, Kalahandi and Rayagada dists.), Tamil Nadu (Chennai dist.), Tripura, Uttarakhand (Dehra Dun, Pauri and Uttarkashi dists.; Gobind Pashu Vihar, Uttarkashi dist.; Rajaji Tiger Reserve, Dehra Dun, Haridwar and Pauri dists.), Uttar Pradesh (Agra and Vanansi dists.; Terai Region) and West Bengal (Bankura, Birbhum, Cooch
Behar, Jalpaiguri, Kolkata, Malda, Midnapur, Nadia, 24 Parganas, Purulia and West Dinajpur dists.; Acharya Jagadish Chandra Bose Indian Botanic Garden / Royal Botanic Garden, Shibpur, Howrah and nr. Kolkata; Buxa Tiger Reserve and Chilapata Forest, Alipurduar dist.).

Elsewhere: Bangladesh, Nepal and Sri Lanka.

Uncertain / possibly: Bhutan, China and Myanmar (Frost, 2021; iucnredlist).

Conservation Status: IUCN Red List- Least Concern.

Remarks: Sarkar and Ray (2006) studied Arunachal Pradesh fauna but didn't report it from there.

\section{Hoplobatrachus tigerinus (Daudin, 1802)}

Rana tigerina Daudin, 1802. An. XI, Hist Nat. Rain. Gren. Crap., Quarto: 64 (type-locality: Bengale); Annandale and Rao, 1918. Rec. Indian Mus., 15 (3): $25-$ 40; Inger and Dutta, 1968. J. Bombay nat. Hist. Soc., 83 (suppl.): 138; Venkateswarlu and Murthy, 1972. Indian J. Zool, 13 (3): 129; Sarkar, 1991. Rec. zool. Surv. India, 89 (1-4): 213, 215; Chanda, 2002. Handbook Indian Amphibians: 265; Sarkar and Ray, 2002. Amphibia. In: Fauna of Kabar Lake. Wetland Ecosystem Series, 4: 110-111; Sarkar et al., 2004. Fauna of Bihar (including Jharkhand), Part I, State Fauna Series, 11: 190-191.

Hoplobatrachus tigerina, Saniyal et al., 2014. Rec. zool. Surv. India, Occ. Paper No. 361:20.

Hoplobatrachus tigerinus, Hussain et al., 2017. An assessment report on biodiversity value of Gautam Buddha Wildlife Sanctuary, Bihar and Jharkhand: 22; Kumar, 2019. frog leg \# 138. In: Zoo's Print, 34 (8):13, 14, fig.; Pankaj, 2020. International Research Journal of Modernization in Engineering Technology and Science, 2 (1): 79, 80, 81; Pankaj and Nath, 2021a. Reptiles and Amphibians, 28 (1): 107, fig. 2F; Pankaj and Nath, 2021b. MOJ Biology and Medicine, 6 (2): 6061.

Common Names: Bull-frog, Golden Frog, Tiger Frog. Diagnostic Features: Interorbital width much smaller than upper eyelid, about $1 / 3^{\text {rd }}$ of eye diameter and less than internarial space; tympanum distinct, nearly $2 / 3^{\text {rd }}$ or equal to eye diameter; fingers free, $1^{\text {st }}$ longer than $2^{\text {nd }}, 3^{\text {rd }}$ longest, $4^{\text {th }}$ almost equal to $2^{\text {nd }}$ or slightly longer, tips rounded (Chanda, 1994) or obtusely pointed (Ray, 1999) or tips not sharply pointed (Sarkar and Ray, 2002, 2006; Deuti et al., 2017); subarticular tubercles small but very prominent (Chanda, 1994) or well developed (Ray, 1999) or small and feebly developed (Chanda, 2002); subarticular tubercles of fingers and toes not very distinct (Sarkar and Ray, 2002, 2006); toes fully webbed, webbing on two distal digits of $4^{\text {th }}$ toe very narrow but connecting tips, with prominent rounded tips, subarticular tubercles small but prominent, a prominent dermal fold on inner side of $5^{\text {th }}$ toe (Chanda, 1994); toes obtuse, somewhat swollen at tip, metatarsals separated nearly to base, subarticular tubercles distinct but comparatively smaller in size (Ray, 1999); toes not sharply pointed (Deuti et al., 
2017); inner metatarsal tubercle blunt (not shovelshaped), elongated and about $2 / 3^{\text {rd }}$ of inner toe, outer metatarsal tubercle absent (Ray, 1999); tibio-tarsal articulation reaching hind end of eye (Ray, 1999) or beyond snout tip (Chanda, 1994, 2002) or reaching between hind edge of eye and nostril (Sarkar et al., 1992; Sarkar and Ray, 2002, 2006); hind limbs when folded at right angles to body, heals overlap.

Colouration: Olive-brown, yellowish-green or olive with irregular dark patches and yellowish vertebral stripe; warts and long glandular folds. Males acquire bright yellow colour during breeding season to attract females.

Snout-Vent Length: 65-158 mm (Boulenger, 1920); 16$161 \mathrm{~mm}$ (Sanyal et al., 1991); 89-137 mm (Sarkar, 1991); 17-154 mm (Sarkar et al., 1992); 71.0-88.5 mm (Chanda, 1994); 62 mm (Chanda, 1995b); 52-143 (Ray and Tilak, 1995); $134 \mathrm{~mm}$ (Chanda, 2002); 20-159 mm (Sarkar et al., 2004); 72-143 mm (Sarkar and Ray, 2006); 54-94 and 50-144 mm (Deuti et al., 2014); 41.22-85.64 and 60-150 mm (Deuti et al., 2017); male $153.9 \mathrm{~mm}$ (Prasad et al., 2020).

Altitudinal Record: Up to $1,800 \mathrm{~m}$ in India (Ray and Tilak, 1995); Up to 1,700 m (Ray, 1999); 2,000 $\mathrm{m}$ at Nepal (Dubois, 1976; Husain, 2015); up to $1,100 \mathrm{~m}$ (Ingle, 2011); $129 \mathrm{~m}$ at Renigunta, Andhra Pradesh; $370 \mathrm{~m}$ at Rayagada, $435 \mathrm{~m}$ at Semiliguda, Odisha (Deuti et al., 2014); 25-800 m (iucnredlist).

\section{Distribution:}

New Record: Erki, Gandhar and Safepur, Jehanabd (Bihar).

Bihar: Aurangabad (Raja Jagganath Dam Deo), Begusarai (Kabar Lake), Bhojpur, East Champaran, Gaya (Gautam Buddha Wildlife Sanctuary), Nalanda (Rajgir Wildlife Sanctuary), Patna and Saran districts.

Jharkhand: Bokaro, East Singhbhum, Gumla, Hazaribagh, Koderma (Gautam Buddha Wildlife Sanctuary); Ranchi, Santhal Parganas and West Singhbhum districts.

Rest of India: Andhra Pradesh (Chittoor dist.), Arunachal Pradesh (East Siang, Lower Subansiri and West Kameng dists), Assam (Goalpara, Golaghat, Kamrup and Sivasagar and Tinsukia dists.), Chhattisgarh (Bastar, Bijapur, Dantewada, Dhamtari, Jashpur, Kanker, Kondagaon, Narainpur and Sukma dists.; Indravati Tiger Reserve, Bijapur dist.; Kanger Valley National Park, Bastar dist.), Delhi (South Delhi dist.), Goa (South Goa dist.), Gujarat (Dang and Patan dists.), Haryana, Himachal Pradesh, Jammu and Kashmir, Karnataka (Madekeri, Shimoga, Shivamogga and Uttara Kannada dists.), Kerala (Alappuzha, Ernakulam, Idukki, Kollam and Pathanamthitta dists.; Malabar), Madhya Pradesh (Balaghat, Dinori, Gwalior, Hoshangabad, Jabalpur, Mandla, Morena, Seoni, Shivpuri and Ujjain dists.; Kanha Tiger Reserve, Balaghat and Mandla dists.; Panna Tiger Reserve, Panna and Chhatarpur dists.; Pench Tiger Reserve, Seoni dist.), Maharashtra (Chandrapur, Kolhapur, Mumbai Suburban, Nagpur, Pune, Raigad, Ratnagiri,
Sangli, Sindhudurg and Thane dists.), Manipur (Churachandpur dist.), Mizoram (Dampa Tiger Reserve, Mamit dist.), Nagaland (Kohima dist.), Odisha (Koraput and Rayagada dists.), Punjab, Rajasthan (Ajmer and Jhalawar dists.; Aravalli), Sikkim, Tamil Nadu, Telangana (Khammam dist.), Tripura (Gomati dist.), Uttarakhand (Dehra Dun, Nainital, Pauri and Tehri dists.; Corbett Tiger Reserve, Nainital and Pauri dists.; Rajaji Tiger Reserve, Dehra Dun, Haridwar and Pauri dists.), Uttar Pradesh, West Bengal (Bankura, Bardhaman, Jalpaiguri, Kolkata, Darjeeling, Kolkata, Malda, Midnapur, Murshidabad, Nadia, Purulia, South 24 Parganas and West Dinajpur dists.; Alipurduar, Buxa Tiger Reserve a Chilapata Forest, Alipurduar dist.; Gorumara National Park, Jalpaiguri dist,) and Eastern and Western Ghats.

Introduced: Andaman and Nicobar Islands (Frost, 2021).

Elsewhere: Afghanistan, Bangladesh, Bhutan, Myanmar, Nepal and Pakistan.

Introduced: Madagascar and Maldives (Frost, 2021; iucnredlist).

Uncertain: China (being belong to $H$. rugulosus, Wiegmann, 1834) (Zhao and Adler, 1993; iucnredlist).

Conservation Status: IUCN Red List- Least Concern.

Remarks: Cannibalism has been observed in this species and also predation on other frogs.

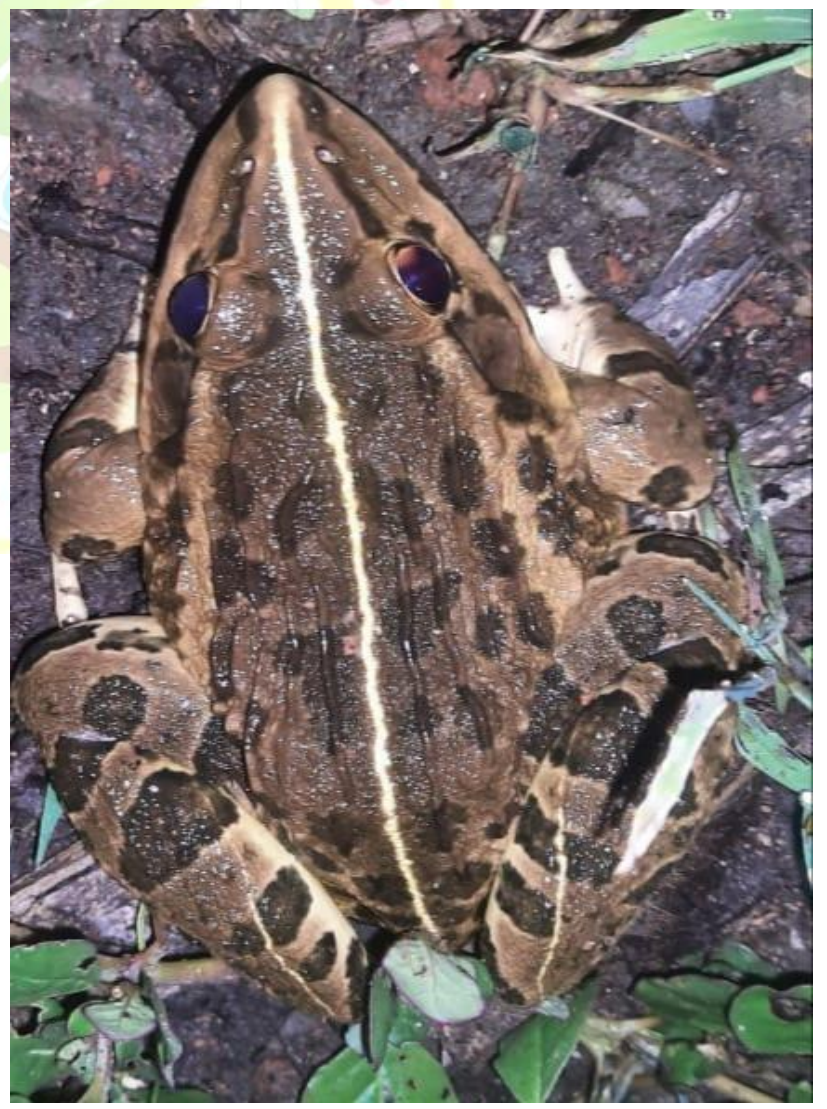

Figure 3a. Hoplobatrachus tigerinus, Common Bullfrog (brown, from Jehanabad, Bihar; Credit: Kumar Sahab) 


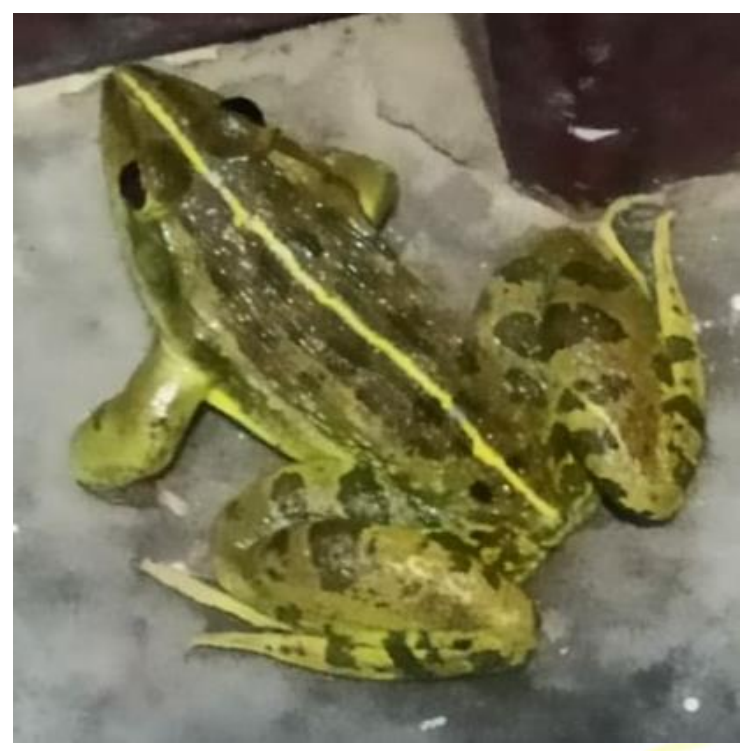

Figure 3b. H. tigerinus, Bull-frog (green, from Jehanabd, Bihar; by $2^{\text {nd }}$ author)

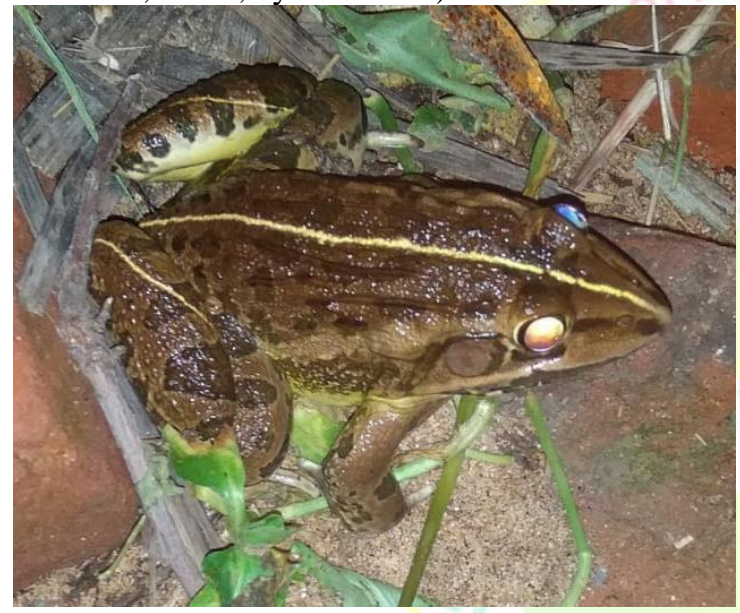

Figure 3c. H. tigerinus, Bull-frog (brown with prominent white line on hind leg and distinct tympanum, from Jehanabd, Bihar; Credit: Mr. Ramlakhan Thakur)

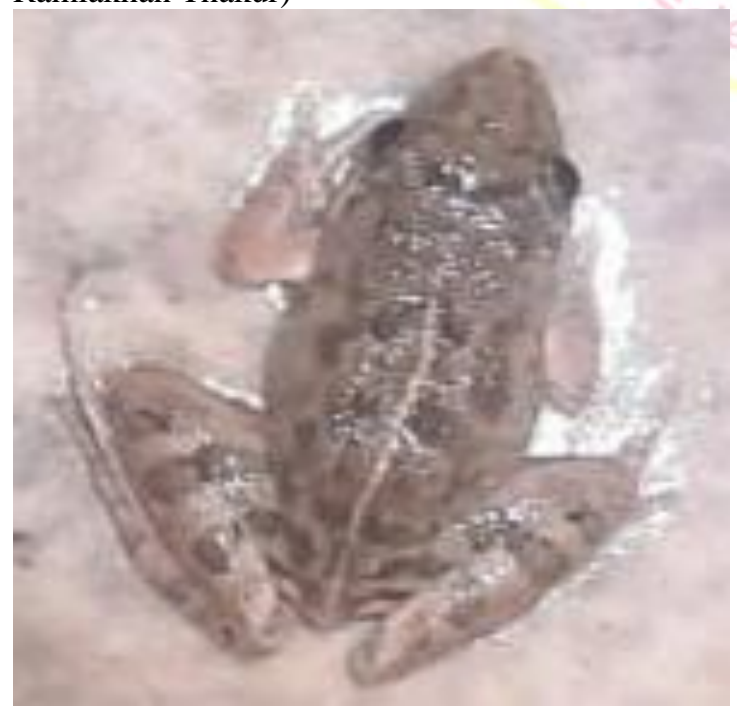

Figure 3d. H. tigerinus, Bull-frog (sub-adult, from Jehanabad, Bihar; Credit: Mr. Ramlakhan Thakur)

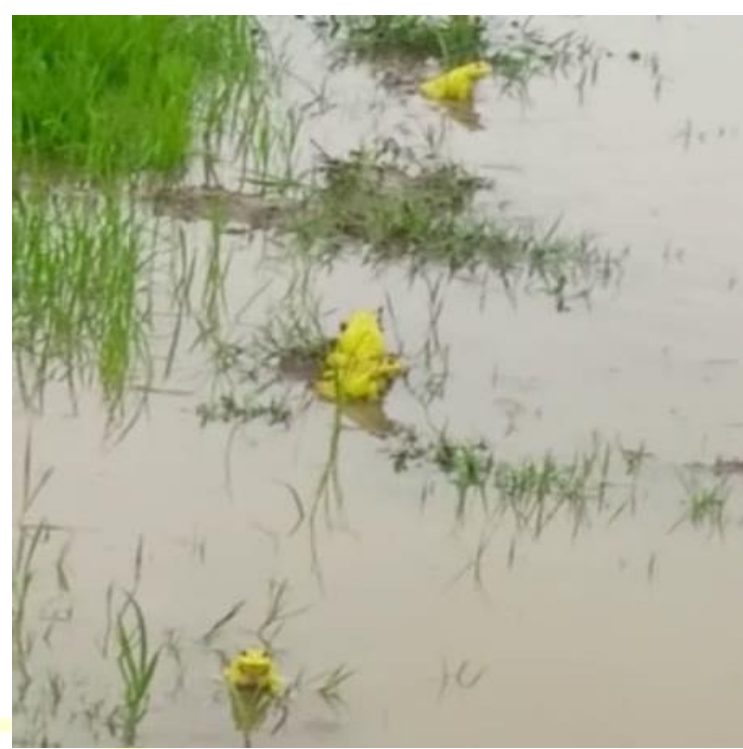

Figure 3e. H. tigerinus, Bull-frog (male in breeding bright yellow colour, from Jehanabad, Bihar; Credit: Mr. Arvind Kumar)

\section{Genus: Sphaerotheca Guenther, 1859}

6. Sphaerotheca breviceps (Schneider, 1799)

Rana breviceps Schneider, 1799. Hist. Amph. Nat., 1: 140 (type-locality; Indes orientales [probablement de Tranquebar, Tamil Nadu; $\left.11^{\circ} 02^{\prime} \mathrm{N}, 79^{\circ} 51^{\prime} \mathrm{E}\right]$ ).

Tomopterna breviceps, Venkateswarlu and Murthy, 1972. Indian J. Zool, 13 (3): 129; Inger and Dutta, 1986. J. Bombay nat. Hist. Soc., 83 (suppl.): 138; Sarkar, 1991. Rec. zool. Surv. India, 89 (1-4): 215-216; Ray, 1999. Mem. zool. Syrv. India, 18 (3): 50-54, pl. 17, figs. 1-6; Chanda, 2002. Handbook Indian Amphibians: 212, 265; Sarkar et al., 2004. Fauna of Bihar (including Jharkhand), Part I, State Fauna Series, 11: 191.

Sphaerotheca breviceps, Saniyal et al., 2014. Rec. zool. Surv. India, Occ. Paper No. 361: 20; Kumar, 2019. frog leg \# 138. In: Zoo's Print, 34 (8):13; Dandekar, et al., 2020. Reptiles and Amphibians, 27 (3): 391, 392, 395figs. 2A; 3 (map), 6; Pankaj, 2020. International Research Journal of Modernization in Engineering Technology and Science, 2 (1): 79, 80, 81; Pankaj and Nath, 2021a. Reptiles and Amphibians, 28 (1): 107, fig. 2G; Pankaj and Nath, 2021b. MOJ Biology and Medicine, 6 (2): 61.

Common Names: Burrowing Frog, Indian Burrowing Frog, Olive Frog, Short-headed Burrowing Frog.

Diagnostic Features: Interorbital width much smaller than upper eyelid; tympanum distinct, vertically oval, about half of eye diameter; fingers free, $1^{\text {st }}$ longer than $2^{\text {nd }}$ and with a large, rounded subarticular tubercle on palmar surface, $3^{\text {rd }}$ finger equal to or slightly longer than $1^{\text {st }}, 4^{\text {th }}$ shortest, subarticular tubercles on $2^{\text {nd }}, 3^{\text {rd }}$ and $4^{\text {th }}$ finger almost equal in size and quite prominent, $1^{\text {st }}$ finger with a large, rounded subarticular tubercle on palm; tips swollen (Sarkar et al., 1992) or blunt (Chanda, 2002); subarticular tubercles large, very prominent and subconical (Chanda, 2002); toes $1 / 2$ or 
slightly webbed, more than three phalanges of $4^{\text {th }}$ toe free; tips obtuse, subarticular tubercles prominent but smaller than those of fingers, inner metatarsal tubercle well developed and shovel-shaped, compressed and placed obliquely at base of $1^{\text {st }}$ toe, outer indistinct, being united with muscles (Ray, 1999), outer metatarsal tubercle absent (Sarkar et al., 2004); tibio-tarsal tubercle distinct, indistinct or absent (Bhaduri and Kripalani, 1954) or absent (in Bokaro and Dhanbad specimens, Sarkar, 1991); tibio-tarsal articulation reaching axil or shoulder (Sarkar et al., 1992; Sarkar et al., 2004) or shoulder (Ray, 1999) or tympanum (Chanda, 2002).

Colouration: Brownish, greyish, olive or yellowish and marbled with dark brown.

Snout-Vent Length: 35-56 mm (Boulenger, 1920); 23$28 \mathrm{~mm}$ (Sanyal et al., 1991); 34-41 mm (Sarkar, 1991); 13-52 mm (Sarkar et al., 1992); 29 mm (Chanda, 1995b); 65 mm (Chanda, 2002); 13-42 (Sarkar et al., 2004); 65 mm (Husain, 2015).

Altitudinal Records: Himalayas up to 2,134 m / 7,000 ft (Annandale and Rao, 1918); 1,500 m (Husain, 2015; iucnredlist).

\section{Distribution:}

Bihar: Aurangabad (Raja Jagannath Dam Deo), Bhojpur and Gaya districts.

Jharkhand: Bokaro, Dhanbad, East Singhbhum, Hazaribagh, Ranchi and West Singhbhum districts.

Rest of India: Andhra Pradesh (Kapada dist.), Assam, Chhattisgarh (Bastar, Bijapur, Dantewada, Kanker, Kondagaon, Narainpur and Sukma dists.; Indravati Tiger Reserve, Bijapur dist.; Kanger Valley National Park, Bastar dist.), Delhi, Eastern Ghats, Gujarat (Dahod, Dang, Rajkot and Saba Kantha dists.), Haryana, Himachal Pradesh (Sirmaur dist.), Jammu and Kashmir, Karnataka (Bengaluru and Coorg/Kodagu dists.), Kerala (Idukki, Malabar and Thiruvananthapuram dists.), Madhya Pradesh (Gwalior, Jabalpur and Morena, Seoni and Ujjain dists.; Pench Tiger Reserve, Seoni dist.), Maharashtra (Mumbai City, Nagpur and Ratnagiri dists.), Odisha, Punjab, Rajasthan (Aravalli Range), Tamil Nadu (Chennai and Mayiladuthurai dists.), Uttarakhand (Dehra Dun, Nainital, Pauri, Pithoragarh, Tehri and Uttarkashi dists.; Corbett Tiger Reserve, Nainital and Pauri dists.; Gobind Pashu Vihar, Uttarkashi dists.; Rajaji Tiger Reserve, Dehra Dun, Haridwar and Pauri dists.), Uttar Pradesh (Agra and Prayagraj / Allahabad dists.) and West Bengal (Bankura, Birbhum, Jalpaiguri, Midnapur, Murshidabad, Nadia, 24 Parganas, Purulia and West Dinajpur dists.).

Doubtful: Sikkim (Subba et al., 2016).

Elsewhere: Myanmar, Nepal, Pakistan and Sri Lanka.

Uncertain: Bangladesh and Maldives.

Conservation Status: IUCN Red List- Least Concern.

Remarks: Frost (2021) mentioned that records from North India and Pakistan are attributable to other species. This needs to be studied.
7. Sphaerotheca magadha Prasad et al., 2019

Sphaerotheca magadha Prasad, Dinesh, Das, Swamy, Shinde and Vishnu, 2019. Rec. zool. Surv. India, 119 (3): 197-210 (type-locality: semi urbanized agricultural land of Nawadih village [N 24.4179; E 85.4680, 380 meters asl], Koderma, Jharkhand); Dandekar, et al., 2020. Reptiles and Amphibians, 27 (3): 390, 392, 394, figs. 1D; 3 (map), 6; Mukherjee, A., 2021. Zoos' Print Journal, 36: 31-32.

Common Name: Magadh Burrowing Frog.

Diagnostic Features: Fore limbs robust and short; fingers short and thin without any dermal fringes, $1^{\text {st }}$ longer than $2^{\text {nd }}$ and sub-equal to $3^{\text {rd }}$, tips blunt and without enlarged discs, webbing absent, subarticular tubercles distinct, rounded; pre-pollex tubercle distinct; supernumerary tubercles absent; inner toe minute; webbing moderate; inner metatarsal tubercle distinct and shovel-shaped, outer tubercle and supernumerary tubercles absent; tarsal tubercle prominent; tibio-tarsal articulation reaching front of shoulder; hind limbs short, when folded at right angles to body, heals not touching each other.

Colouration: Light greenish-brown marbled with dark, with raised glandular blackish folds, male with a pair of black vocal sacs.

Snout-Vent Length: 28.5-34.2 mm (Prasad et al., 2019).

Distribution: Endemic to India.

Bihar: No record.

Jharkhand: Koderma and Maithon districts (Chota Nagpur Plateau).

Rest of India: Madhya Pradesh (Panna Tiger Reserve, Panna and Chhatarpur dists.), Maharashtra (Chandrapur dist.) and West Bengal (Purulia dist.).

Elsewhere: No record.

Conservation Status: IUCN Red List- Data Deficient.

\section{Sphaerotheca rolandae (Dubois, 1983)}

Rana (Tomopterna) breviceps rolandae Dubois, 1983, Alytes, 2: 166 (type-locality: Kurunegala, Sri Lanka, altitude $60 \mathrm{~m}$, latitude $7^{\circ} 29^{\prime} \mathrm{N}$, longitude $80^{\circ} 22^{\prime} \mathrm{E}$ ).

Sphaerotheca rolandae, Hedge and Roy, 2011. frog leg \# 17: 20-21; Kumar, 2019. frog leg \# 138. In: Zoo's Print, 34 (8):13, 15, fig.; Pankaj and Nath, 2021a. Reptiles and Amphibians, 28 (1): 107; Pankaj and Nath, 2021b. MOJ Biology and Medicine, 6 (2): 61.

Common Names: Marble Sand Frog, Roland's Burrowing Frog, Southern Burrowing Frog.

Diagnostic Features: Interorbital space broader than upper eyelid; tympanum distinct, nearly half of eye diameter; fingers free, $1^{\text {st }}$ much longer than $2^{\text {nd }}$, tips swollen, subarticular tubercles small, distinct; outer meta-tarsal tubercle prominent, shovel-shaped, larger than $1^{\text {st }}$ toe, outer absent; tibio-tarsal articulation reaching hind edge of eye and its tubercle prominent.

Colouration: Dorsum uniformly grey, may be indistinctly marbled with dark brown; limbs with or without darker cross bands; thigh black spotted above or marbled with white; skin granulated; strongly curved 
fold between eye and shoulder; vocal sac subgular area blackish.

Snout-Vent Length: Up to $35 \mathrm{~mm}$ (Srinivasulu et al., 2006).

Altitudinal Records: $60 \mathrm{~m}$ at Kurunegala, Sri Lanka (Dubois, 1983); up to $200 \mathrm{~m}$ (iucnredlist).

Distribution:

Bihar: Gaya and Nalanda districts.

Jharkhand: No record.

Rest of India: Andhra Pradesh (NagarjunasagarSrisailam Tiger Reserve, Guntur, Kurnool, Nalgonda and Prakasam dists.), Chhattisgarh (Bastar, Bijapur, Dantewada, Kanker, Kondagaon, Narainpur and Sukma dists.; Indravati Tiger Reserve, Bijapur dist.; Kanger Valley National Park, Bastar dist.), Kerala, Madhya Pradesh, Odisha, Rajasthan (Aravalli), Tamil Nadu, Telangana (Nagarjunasagar-Srisailam Tiger Reserve, Mahabubnagar dist.), Uttar Pradesh (Ballia dist.) and West Bengal.

Elsewhere: Sri Lanka.

Conservation Status: IUCN Red List- Least Concern.

Remarks: Frost (2021) considered it a Sri Lankan form. Dutta (1997), Biju (2001) and Chanda (2002) recorded it from Indian localities (Madhya Pradesh, Kerala, Odisha, Tamil Nadu and West Bengal) and followed by Dinesh et al. (2009). Its distribution has also been shown in India and Sri Lanka in the IUCN Red List.

Subfamily: Occidozyginae Fei, Ye and Huang, 1990 Genus: Occidozyga Kuhl and Van Hasselt, 1822

9. Occidozyga lima (Gravenhorst, 1829)

Rana lima Gravenhorst, 1829, Delic. Mus. Zool. Vratislav., 1: 41 (type locality: Java).

Occidozyga lima, Hussain et al., 2017. An assessment report on biodiversity value of Gautam Buddha Wildlife Sanctuary, Bihar and Jharkhand: 22.

Common Names: Green Floating Frog, Green Puddle Frog, Pointed-tongue Floating Frog, Rough-skinned Floating Frog.

Diagnostic Features: Interorbital with shorter than upper eyelid; tympanum indistinct, equal to eye; fingers free $1^{\text {st }}$ equal to $2^{\text {nd }}$, tips pointed; subarticular tubercles minute; toes fully webbed, tips swollen; inner metatarsal tubercle shovel-shaped, outer sub-conical; a small tarsal tubercle just below tibio-tarsal articulation which reaches middle of eye to snout tip.

Colouration: Brownish, greyish or olive-brown with a broad greenish vertebra stripe and small warts.

Snout-Vent Length: male 39, female $40 \mathrm{~mm}$ (ecologyasia).

Altitudinal Record: Upper limit 750 m (iucnredlist).

Distribution:

Bihar: Gaya (Gautam Buddha Wildlife Sanctuary) district.

Jharkhand: Koderma (Gautam Buddha Wildlife

Sanctuary) district.

Rest of India: West Bengal.
Elsewhere: Bangladesh, Cambodia, China, Hong Kong, Indonesia, Laos, Malaysia, Myanmar, Thailand and Viet Nam.

Uncertain: Nepal (iucnredlist; wikipedia).

Conservation Status: IUCN Red List- Least Concern

FAMILY: MICROHYLIDAE GUENTHER, 1858

Subfamily: Microhylinae Guenther, 1858

Genus: Kaloula Gray, 1831

10. Kaloula assamensis Das et al., 2005

Kaloula assamensis Das, Sengupta, Ahmed and Dutta, 2005, Hamadryad, 29: 103 (101-109) (type-locality: Majbat $\left(26^{\circ} 45^{\prime} \mathrm{N}, 92^{\circ} 20^{\prime} \mathrm{E}\right.$; $141 \mathrm{~m}$ ASL, Udalguri dist. and Sirajuli $\left(26^{\circ} 4^{\prime} \quad \mathrm{N}, 92^{\circ} 1^{\prime} 2^{\prime}\right.$ E), Nameri Wildlife Sanctuary ( $\left.26^{\circ} 56^{\prime} \mathrm{N}, 92^{\circ} 52^{\prime} \mathrm{E}\right)$, Sonitpur dist.; Orang National Park (26 $\left.36^{\circ} \mathrm{N}, 92^{\circ} 15^{\prime} \mathrm{E}\right)$, Darrang dist., Assam and Pakhui Wildlife Sanctuary $\left(26^{\circ} 55^{\prime} \mathrm{N}, 92^{\circ} 51^{\prime} \mathrm{E}\right)$ in East Kameng dist., Arunachal Pradesh); Nath et al., 2011. frog leg \# 17: 22-23; Talukder et al., 2007. Cobra, 1 (1): 18-20; Paul et al., 2007. ibid., 1 (3): 15-16; Sengupta et al., 2009. Nat. Hist. Jour. Chulalongkorn University, 9 (2): 209-222; Deuti et al., 2012. J. Bombay nat. Hist. Soc., 109 (3): 208-209.

Kaloula pulchra, Ray, 1998 (nec Gray, 1831). Amphibia. In: Fauna of Valmiki Tiger Reserve. Fauna of Conservation Area, No. 10: 45-46.

Common Names: Assam Balloon Frog, Assam Kaloula, Assam Narrow-mouth Frog, Assam Painted Frog.

Diagnostic Features: Head small, broader than long, snout rounded; eyes large; interorbital region flat; a distinct supra-tympanic fold extending from eye to base of fore leg; fingers free, tips slightly dilated; toes halfwebbed, tips slightly rounded, webbing reaching tips of all toes as a narrow sheath, except $4^{\text {th }}$ toe where it is up to medial tubercle; pointed inner and outer pedal tubercles (Deuti et al., 2017).

Colouration: Pale brownish with dark-edged bright yellow vertebral stripe and two black-edged broad dark brown lateral patches between eye and groin.

Snout-Vent Length: $35 \mathrm{~mm}$ (Ray, 1998, as K. pulchra); $30.7 \mathrm{~mm}$ (Deuti et al., 2012); 30-43 mm (Deuti et al., 2017).

Altitudinal Record: 48-141 m (iucnredlist).

Distribution:

Bihar: Manguraha (27 $\left.28^{\prime} \mathrm{N} ; 8^{\circ} 12^{\prime} \mathrm{E}\right)$, Valmiki Tiger

Reserve, West Champaran district.

Jharkhand: No record.

Rest of India: Arunachal Pradesh (Pakke / Pakhui Wildlife Sanctuary, East Kameng dist.), Assam (Bongaigaon and Udalguri dists.; Orang National Park, Darrang and Sonitpur dists.; Nameri National Park, Sonitpur dist.) and West Bengal (Alipurduar and Cooch Behar dists.).

Elsewhere: No record (Sen and Mathew, 2008).

Uncertain: Bangladesh (iucnredlist).

Conservation Status: IUCN Red List- Least Concern. 
Remarks: The specimen collected from Manguraha $\left(27^{\circ} 28^{\prime} \mathrm{N} ; 8^{\circ} 12^{\prime} \mathrm{E}\right)$, Valmiki Tiger Reserve, West Champaran district (Bihar), was misidentified and reported as K. pulchra by Ray (1998). It was restudied and identified as $K$. assamensis Das et al. 2005 by Deuti et al. (2012) on its smaller size $(30.7 \mathrm{~mm})$, presence of a bright yellow vertebral stripe and broad dark brown lateral stripes on either side where as $K$. pulchra is larger in size $(35-55 \mathrm{~mm})$ and differs in the presence of several black-edged reddish-brown irregular blotches above and in absence of the lateral stripes.

\section{Kaloula pulchra Gray, 1831}

Kaloula pulchra Gray, 1831. Zool. Misc., 1: 38 (typelocality: China); Vankateswarlu and Murthy, 1972. Indian J. Zool., 13 (3): 130; Sarkar et al., 2004. Fauna of Bihar (including Jharkhand), Part I, State Fauna Series, 11: 185; Deuti et al., 2012. J. Bombay nat. Hist. Soc., 109 (3): 209.

Common Names: Banded Bull-frog, Painted Balloon Frog, Painted Frog, Painted Bull-frog, Painted Burrowing Frog, Painted Kaloula.

Diagnostic Features: Interorbital width much broader than upper eyelid, one and half to twice eye diameter; tympanum hidden; fingers moderately long, free, $1^{\text {st }}$ finger shorter than $2^{\text {nd }}$; tips dilated into well developed truncated discs (Sarkar et al., 1992); subarticular tubercles of fingers and toes distinct; toes short, $1 / 3^{\text {rd }}$ or slightly webbed at base; tips obtusely swollen (Sarkar et al., 1992); inner metatarsal tubercles large and shovelshaped, outer small, also shovel-shaped; tibio-tarsal articulation reaching shoulder or slightly beyond (Chanda, 2002) or axilla (Sarkar et al., 1992; Sarkar et al., 2004).

Colouration: Pink, brown or dark brown, with a large irregular yellowish-brown marking and narrow dark lateral stripe from behind eye almost to the groin, separated from mid-dorsal dark brown coloration by a yellow dorso-lateral stripe, which may itself be punctuated with several spots, with scattered warts; belly mottled dirty yellowish brown; males with a black throat.

Snout-Vent Length: 13-58 mm (Sarkar et al., 1992); 80$85 \mathrm{~mm}$ (Chanda, 2002); $27 \mathrm{~mm}$ (Sarkar et al., 2004); 45-85 mm (Deuti et al., 2012); 54-70 mm male, 57-75 $\mathrm{mm}$ females (amphibiaweb).

Altitudinal Record: Up to 750 m (iucnredlist).

\section{Distribution:}

Bihar: Vaishali (Bhagwatpur) and West Champaran district.

Jharkhand: No record.

Rest of India: Assam (Chachar and Karimganj dists.), Karnataka, Kerala, Madhya Pradesh, Maharashtra (Palghar dist.), Manipur, Meghalaya, Mizoram, Nagaland, Odisha, Tamil Nadu, Tripura (North Tripura dist.) and West Bengal (Birbhum, Cooch Behar, Hooghly, Nadia, North Dinajpur, 24 Parganas, Purulia and West Dinajpur dists.).
Elsewhere: Bangladesh, Brunei, Cambodia, China, Hong Kong, Indonesia, Laos, Malaysia, Myanmar, Singapore, Sri Lanka, Thailand and Viet Nam.

Incidental: Guam (Christy et al., 2007).

Introduced: Philippines (Siler et al., 2011).

Uncertain: Nepal (Shah and Tiwari, 2004).

Conservation Status: IUCN Red List- Least Concern.

\section{Genus Microhyla Tschudi, 1838}

12. Microhyla ornata (Dumeril and Bibron, 1841)

Engystoma ornatum Dumeril and Bibron, 1841. Erp. Gen., 8: 745 (type-locality: cote Malabar).

Microhyla ornata, Inger and Dutta, 1968. J. Bombay nat. Hist. Soc., 83 (suppl.): 136; Vankateswarlu and Murthy, 1972. Indian J. Zool., 13 (3): 130; Sarkar, 1991. Rec. zool. Surv. India, 89 (1-4): 212-213; Chanda, 2002. Handbook Indian Amphibians: 265; Sarkar and Ray, 2002. Amphibia. In: Fauna of Kabar Lake. Wetland Ecosystem Series, 4: 108; Sarkar et al., 2004. Fauna of Bihar (including Jharkhand), Part I, State Fauna Series, 11: 187; Saniyal et al., 2014. Rec. zool. Surv. India, Occ. Paper No. 361:20; Kumar, 2019. Frog leg \# 138. In: Zoo's Print, 34 (8):13, 15-16, fig.; Pankaj, 2020. International Research Journal of Modernization in Engineering Technology and Science, 2 (1): $79,80,81$.

Common Names: Ornate Narrow-mouthed Frog, Ornate Rice Frog, Ornamented Pygmy Frog.

Diagnostic Features: Interorbital width a slightly broader than upper eyelid; tympanum hidden; fingers free, $1^{\text {st }}$ shorter than $2^{\text {nd }}, 3^{\text {rd }}$ longest, $4^{\text {th }}$ slightly longer

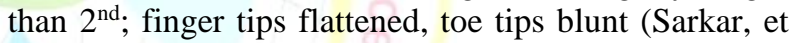
al., 1992) or tips of fingers and toes swollen into a small round knob on ventral side (Ray, 1999) or dilated into very small discs (Chanda, 1995a, 2002) or flattened and blunt but without enlarged discs (Deuti et al., 2017); subarticular tubercles small, distinct (Chanda, 1995a); two elongated palmer tubercles; toes with rudimentary webbing; subarticular tubercles small, distinct; inner metatarsal tubercle elongated, outer small and rounded (Chanda, 2002); outer metatarsal tubercle absent (Chanda, 1994, 1995a); two small but distinct oval and outer pedal tubercles (Deuti et al., 2017); tibio-tarsal articulation reaching near eye (Sarkar, et al., 1992) or slightly in front of shoulder (Chanda, 1995a) or between shoulder and eye (Chanda, 2002); legs when folded at right angles to body, heals overlap.

Colouration: Brownish, bronze, reddish or greenishbrown with large dark brown markings which may be diamond-shaped.

Snout-Vent Length: 11-22 mm (Sanyal et al., 1991); 13-21 mm (Sarkar, 1991); 9-25 mm (Sarkar et al., 1992); 22-25 mm (Chanda, 1994); 12-20 mm (Chanda, 1995b); 7-20 mm (Ray and Tilak, 1995); $30 \mathrm{~mm}$ (Chanda, 2002); 9-21 mm (Sarkar et al., 2004); average $25 \mathrm{~mm}$ (Ingle, 2011); 18-24 and 17-26 mm (Deuti et al., 2014); $20 \mathrm{~mm}$ (Dutta, 2015); $28 \mathrm{~mm}$ (Husain, 2015); 18-27 mm (Deuti et al., 2017); 15-25 mm (Ingle, 2020). 
Altitudinal Records: 1,524 m (Sanyal et al., 1991; Sarkar, 1991; Chanda, 1995a; Sarkar and Ray, 2002; Chanda, 1995, 2002); $129 \mathrm{~m}$ at Renigunta, Andhra Pradesh; $785 \mathrm{~m}$ at Karlapat, $895 \mathrm{~m}$ at Damanjodi and $355 \mathrm{~m}$ at Muniguda, Odisha (Deuti et al., 2014); 2,000 m (Husain, 2015; iucnredlist).

\section{Distribution:}

Bihar: Aurangabad (Raja Jagannath Dam Deo), Begusarai (Kabar Lake), East Champaran and Nalanda (Rajgir Wildlife Sanctuary) district.

Jharkhand: Bokaro, East Singhbhum, Dhanbad, Hazaribagh, Ranchi and West Singhbhum districts.

Rest of India: Andaman and Nicobar Islands, Andhra Pradesh (Chittoor dist.), Assam (Goalpara dist.; Kaziranga National Park, Golaghat, Karbi Anglong and Nagaon dists.), Chhattisgarh (Bastar, Bijapur, Bilaspur, Dantewada, Jashpur, Kanker, Kondagaon, Narainpur and Sukma dists.; Indravati Tiger Reserve, Bijapur dist.; Kanger Valley National Park, Bastar dist.), Delhi, Eastern Ghats, Goa (South Goa dist.), Gujarat, Haryana, Himachal Pradesh, Jammu and Kashmir, Karnataka (Mysore dist.), Kerala, Madhya Pradesh (Gwalior, Jabalpur, Mandla, Morena, Seoni, Shivpuri and Ujjain dists.; Kanha Tiger Reserve, Balaghat and Mandla dists.; Pench Tiger Reserve, Seoni dist.), Maharashtra (Chandrapur, Kolhapur, Pune, Sangli, Sindhudurg and Solapur dists.), Manipur, Meghalaya (East Khasi Hills, North Garo Hills and West Garo Hills dists.), Mizoram (Serchhip dist.), Nagaland (Kohima dist.), Odisha (Koraput and Rayagada dists.; Karlapat Wildlife Sanctuary, Kalahandi dist.); Punjab, Rajasthan (Aravalli Range), Tripura (Dhalai dist.), Uttarakhand (Dehra Dun, Nainital, Pauri and Tehri dists.; Corbett Tiger Reserve, Nainital and Pari dist.; Rajaji Tiger Reserve, Dehra Dun, Haridwar and Pauri dists.), West Bengal (Bankura, Barddhaman, Jalpaiguri, Kolkata, Malda, Midnapore, Murshidabad, 24 Parganas, Purulia and West Dinajpur dists.; Buxa Tiger Reserve, Chilapata Forest and Jaldapara National Park, Alipuduar dist.) and Western Ghats.

Elsewhere: Bangladesh, Bhutan, China, Indonesia, Japan, Malaysia, Myanmar, Nepal, Pakistan, Sri Lanka, Taiwan, Thailand and Viet Nam.

Conservation Status: IUCN Red List- Least Concern.

\section{Microhyla rubra (Jerdon, 1854)}

Engystoma rubrum Jerdon, 1853, J. Asiat. Soc. Bengal, 22: 534 (type-locality: the Carnatic and Ceylon [southern India and Sri Lanka]).

Microhyla rubra, Kumar, 2019. frog leg \# 138. In: Zoo's Print, 34 (8):13, 15, fig.

Common Names: Densely Spotted Microhylid Frog, Guangdong Rice Frog, Jerdon's Narrow-mouthed Frog, Red Narrow-mouthed Frog.

Diagnostic Features: Interorbital space broader than upper eyelid; tympanum hidden; fingers free, $1^{\text {st }}$ much shorter than $2^{\text {nd }}$, tips rounded (Chanda, 1994, 2002); toes less than half of nearly $1 / 3^{\text {rd }}$ webbed (Chanda, 1994, 2002); tips of fingers and toes not swollen
(Boulenger, 1890); subarticular tubercles distinct; metatarsal tubercles shovel-shaped, inner crescentic, outer semicircular (Chanda, 1940, 2002); tibio-tarsal articulation reaching somewhat beyond shoulder, never to eye (Boulenger, 1890) or eye (Chanda, 1994, 2002); legs when folded at right angles to body, heels overlap (Chanda, 1994).

Colouration: Reddish-brown with a median pinkishbrown band.

Snout-Vent Length: 28-30 mm (Chanda, 1994); $26 \mathrm{~mm}$ (Chanda, 2002); 21-28 and 21-29 mm (Deuti et al., 2014); $30.5 \mathrm{~mm} / 1.2$ in (inaturalist; wikipedia).

Altitudinal Records: $129 \mathrm{~m}$ at Renigunta, Andhra Pradesh (Deuti et al., 2014); up to $700 \mathrm{~m}$ in India and $460 \mathrm{~m}$ in Sri Lanka (iucnredlist).

\section{Distribution:}

Bihar: Nalanda (Rajgir, Pant Wildlife Sanctuary) district.

Jharkhand: No record.

Rest of India: Andhra Pradesh (Chittoor dist.), Assam (Kaziranga National Park, Golaghat, Karbi Anglong and Nagaon dists.), Goa, Karnataka, Kerala, Maharashtra, Tamil Nadu and West Bengal.

Elsewhere: Bangladesh.

Doubtful: Assam, Mizoram, Nagaland, Myanmar and Nepal (Garg et al., 2019).

Uncertain: Goa (Frost, 2021).

Conservation Status: IUCN Red List- Least Concern.

Remarks: Meegaskumbura et al. (2016) distinguished Sri Lankan population from typical M. rubra described from southern India, on the basis of combination of various characters (body size, skin texture and feet dimensions) and recognized Sri Lankan population as a new species, M. mihintalie sp. nov.

\section{Genus: Uperodon Dumeril and Bibron, 1841}

\section{Uperodon globulosus (Guenther, 1864)}

Cocopus globulosus Guenther, 1864. Rept. Br. India: 416 (type-locality: Russelconda [Odisha]).

Uperodon globulosus, Vankateswarlu and Murthy, 1972. Indian J. Zool., 13 (3): 130; Inger and Dutta, 1986. J. Bombay nat. Hist. Soc., 83 (suppl.): 137; Sarkar, 1991. Rec. zool. Surv. India, 89 (1-4): 212; Chanda, 2002. Handbook Indian Amphibians: 46-49, 265; Sarkar et al., 2004. Fauna of Bihar (including Jharkhand), Part I, State Fauna Series, 11: 186; Saniyal et al., 2014. Rec. zool. Surv. India, Occ. Paper No. $361: 19$.

Common Names: Balloon Frog, Globular Frog.

Diagnostic Features: Interorbital width about thrice of upper eyelid width; tympanum hidden; fingers free, $1^{\text {st }}$ shorter than $2^{\text {nd }}, 3^{\text {rd }}$ longest; tips of fingers and toes rounded, without any discs; subarticular tubercles of fingers and toes not distinct; toes with rudimentary webbing; metatarsal tubercles shovel-shaped, inner large, outer smaller; tibio-tarsal articulation not reaching shoulder (Sarkar et al., 1992; Chanda, 2002); hind limbs when folded at right angles to body, heals not touching each other. 
Colouration: Greyish, reddish-brown or golden-brown, speckled with tiny golden or creamy spots; throat of calling male black.

Snout-Vent Length: $68 \mathrm{~mm}$ (Sarkar, 1991); 66-81 mm (Sarkar et al., 1992); 52-54 mm (Chanda, 1994); $50 \mathrm{~mm}$ (Chanda, 1995); 56-61 mm (Sarkar et al., 2004); male 40-64, female 60-84 mm (Deuti et al., 2014); 40-76 mm (Deuti et al., 2017); male 51.9, sub-adult (sex not known) $36.9 \mathrm{~mm}$ (Prasad et al., 2020).

Altitudinal Records: $785 \mathrm{~m}$ at Karlapat and $370 \mathrm{~m}$ at Rayagada, Odisha (Deuti et al., 2014).

\section{Distribution:}

Bihar: (Inger and Dutta, 1986; Chanda, 2002; Dinesh et al., 2009; Sen and Mathew, 2008).

Jharkhand: Bokaro, Chota Nagpur, Dhanbad and Hazaribagh and Jamtara (Gaichand, Rajpalli Jamtara) districts.

Rest of India: Andhra Pradesh, Assam (Jhalukbari and Manas Wildlife Sanctuary, Kamrup dist.), Chhattisgarh (Bastar, Bijapur, Dantewada, Kanker, Kondagaon, Narainpur and Sukma dists.; Indravati Tiger Reserve, Bijapur dist.; Kanger Valley National Park,Bastat dist.), Gujarat, Karnataka (Balgaum dist.), Kerala, Madhya Pradesh (Balaghat and Jabalpur dists.; Panna Tiger Reserve, Panna and Chhatarpur dists.), Maharashtra (Kolhapur, Pune, Sangli and Sindhudurg dists.), Odisha (Rayagada dist.; Karlapat Wildlife Sanctuary, Kalahandi dist.) and West Bengal (Hawrah, Bardhaman, Hooghly, Jalpaiguri, Midnapur and 24 Parganas dists.; Buxa Tiger Reserve and Kodal Basti Forest, Alipurduar dist.).

Elsewhere: Nepal and Sri Lanka.

Uncertain: Bhutan, China and Myanmar (iucnredlist).

Conservation Status: IUCN Red List- Least Concern.

\section{Uperodon systoma (Schneider, 1799)}

Rana systoma Schneider, 1799, Hist. Amph. Nat., 1: 144 (type-locality: India orientali).

Uperodon systoma, Venkateswarlu and Murthy, 1972. Indian J. Zool, 13 (3): 130; Sarkar et al., 2004. Fauna of Bihar (including Jharkhand), Part I, State Fauna Series, 11: 186; Saniyal et al., 2014. Rec. zool. Surv. India, Occ. Paper No. 361:20; Hussain et al., 2017. An assessment report on biodiversity value of Gautam Buddha Wildlife Sanctuary, Bihar and Jharkhand: 22; Kumar, 2019. frog leg \# 138. In: Zoo's Print, 34 (8):13; Sharma and Kumar, 2020. frog leg \# 140. In: Zoo's Print, 35 (9): 18; Pankaj and Nath, 2021a. Reptiles and Amphibians, 28 (1): 107; Pankaj and Nath, 2021b. MOJ Biology and Medicine, 6 (2): 61.

Common Names: Lesser Balloon Frog, Marbled Balloon Frog.

Diagnostic Features: Interorbital space twice as broad as upper eyelid; tympanum hidden; fingers free, $1^{\text {st }}$ shorter than $2^{\text {nd }}$, tips simple, $1^{\text {st }}$ shorter than $2^{\text {nd }}$ which almost equal to $4^{\text {th }}, 3^{\text {rd }}$ longest; subarticular tubercles of fingers and toes indistinct; toes webbed at base or scarcely, tips rounded / simple, $1^{\text {st }}$ toe smallest, $4^{\text {th }}$ longest; metatarsal tubercles strong, compressed, shovel-shaped, inner being very large and as long as $2^{\text {nd }}$ toe, outer large and shovel-shaped, tibio-tarsal articulation reaching shoulder (Chanda, 2002).

Colouration: Pinkish-brown, marbled with dark or blackish-brown reticulated markings.

Snout-Vent Length: 23-46 mm (Ray and Tilak, 1995); $55 \mathrm{~mm}$ (Chanda, 2002); 56-61 mm (Sarkar et al., 2004); 19-52 and 30-55 mm (Deuti et al., 2014); $74 \mathrm{~mm}$ (Husain, 2015); $45 \mathrm{~mm}$ (Ingle, 2020); two individuals (sex not known) 44.6-46.5 mm (Prasad et al., 2020).

Altitudinal Records: 1,000 m at Rajaji Tiger Reserve, Uttarakhand (Ray and Tilak, 1995); up to $1,500 \mathrm{~m}$ (Husain, 2015); $785 \mathrm{~m}$ at Karlapat and $467 \mathrm{~m}$ at Sunabeda, Odisha (Deuti et al., 2014); 1,000 m (iucnredlist).

\section{Distribution:}

Bihar: Gaya (Gautam Buddha Wildlife Sanctuary) district.

Jharkhand: Bokaro, Koderma (Gautam Buddha Wildlife Sanctuary) and East Singhbhum (Dalma Wildlife Sanctuary, Jamshedpur) districts.

Rest of India: Andhra Pradesh (Chittoor dist.), Chhattisgarh (Bastar, Bijapur, Bilaspur, Dantewada, Kanker, Kondagaon, Narainpur and Sukma dists.; Indravati Tiger Reserve, Bijapur dist.; Kanger Valley National Park, Bastar dist.), Gujarat (Sabar Kantha dist.), Himachal Pradesh (Sirmaur dist.), Karnataka, Kerala, Madhya Pradesh (Gwalior dist.; Panna Tiger Reserve, Panna and Chhatarpur dists.), Maharashtra (Aurangabad, Pune, Sangli, Satara and Solapur dists.), Odisha (Koraput dist.; Karlapat Wildlife Sanctuary, Kalahandi dist.), Rajasthan (Jhalawar and Sawai Madhopur dists.; Aravalli Range), Tamil Nadu (Kanchipuram Urban, Tirunelveli and Viluppuram dists.), Uttarakhand (Dehra Dun and Pauri dists.; Corbett Tiger Reserve, Nainital and Pauri dist.; Rajaji Tiger Reserve, Dehra Dun, Haridwar and Pauri dists.), Uttar Pradesh (Agra, Saharanpur and Prayagraj / Allahabad dists.) and West Bengal (Bankura dist.).

Elsewhere: Nepal, Pakistan and Sri Lanka.

Uncertain: Bangladesh (iucnredlist).

Conservation Status: IUCN Red List- Least Concern.

\section{Uperodon taprobanicus (Parker, 1934)}

Kaloula pulchra taprobanica Parker, 1934. Monogr.

Frogs Fam. Microhylidae: 86 (type-locality: Punduloya, Ceylon, 4,000 ft.).

Kaloula taprobanica, Deuti et al., 2012. J. Bombay nat. Hist. Soc., 109 (3): 209; Deuti et al., 2014. Rec. zool. Surv. India, 114 (Part-1): 125-126.

Uperodon taprobanicus, Sharma and Kumar, 2020. frog leg \# 140. In: Zoo's Print, 35 (9): 18.

Common Names: Painted Balloon Frog, Painted Frog, Painted Globular Frog, Painted Kaloula.

Diagnostic Features: Stout-bodied and short-legged; head broader than long, snout rounded, nostrils nearer snout tip than to eye; tympanum hidden; fingers free, tips with well-developed short triangular discs; toes about $1 / 3^{\text {rd }}$ webbed, tips with dilated triangular discs; 
inner metatarsal / pedal tubercle well-developed, large and shovel-shaped, outer small (Deuti et al., 2017).

Colouration: Dark brown with deep red with irregular black-edged pink patches, forming a dark reddish lateral stripe between eye and groin.

Snout-Vent Length: $40 \mathrm{~mm}$ (Daniel, 2002); 35-55 mm (Deuti et al., 2012); 34.01-52.93 and 27-58 mm (Deuti et al., 2014); $58 \mathrm{~mm}$ (Dutta, 2015); 28-58 mm (Deuti et al., 2017).

Altitudinal Records: $785 \mathrm{~m}$ at Karlapat and $467 \mathrm{~m}$ at Sunabeda, Odisha (Deuti et al., 2014); up to $200 \mathrm{~m}$ in India and 1,300 $\mathrm{m}$ in Sri Lanka (iucnredlist).

\section{Distribution:}

New Record: Gandhar, Jehanabad (Bihar).

Bihar: (Dinesh et al., 2009).

Jharkhand: East Singhbhum (Dalma Wildlife Sanctuary, Jamshedpur) district.

Rest of India: Arunachal Pradesh, Assam, Chhattisgarh (Bastar, Bijapur, Dantewada, Kanker, Kondagaon, Narainpur and Sukma dists.; Indravati Tiger Reserve, Bijapur dist.; Kanger Valley National Park, Bastar dist.), Goa, Gujarat, Karnataka (Bengaluru Urban and Ramanagara dists.), Kerala (Ernakulam, Kannur, Kottayam and Thrissur dists.), Madhya Pradesh (Jabalpur, Seoni and Shahdol dists.; Bandhavgarh Tiger Reserve, Umaria dist.; Kanha Tiger Reserve, Balaghat and Mandla dists.; Pench Tiger Reserve, Seoni dist.), Maharashtra (Gondia, Jalgaon and Palghar dists.), Meghalaya, Odisha (Koraput dist.; Karlapat Wildlife Sanctuary, Kalahandi dist.), Tamil Nadu (Chennai dist.), Telangana (Eastern Ghats) and West Bengal (Howrah, Jalpaiguri, Kolkata, Midnapor, Paschim Bardhaman, Purba Bardhaman and South 24 Parganas dist.; Gorumara National Park, Jalpaiguri dist.; Jaldapara National Park, Alipurduar dist.).

Elsewhere: Bangladesh, Nepal and Sri Lanka.

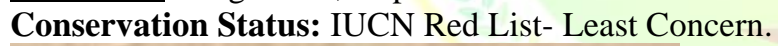

Figure 4. Uperodon taprobanicus, Painted Balloon Frog (from Jehanabd, Bihar; Credit: Mr. Ramlakhan Thakur)
Remarks: Sen and Mathew (2008) doubted its occurrence in N. E. India. However, Dinesh et al. (2009) showed its distribution in Assam, Meghalaya and West Bengal, the north-eadtern states, following Dutta (1997). Frost (2021) also mentioned the distribution in north-eastern India (Arunachal Pradesh, Assam and West Bengal).

\section{Uperodon variegatus (Stoliczka, 1872)}

Callula variegata Stoliczka, 1872. Proc. Asiat. Soc. Bengal, 1872: 111 (type-locality: Ellore [= Eluru], Andhra Pradesh, India. ZSI 2761designated lectotype by Garg et al., 2018).

Ramanella variegata, Sarkar, 1991. Rec. zool. Surv. India, 89 (1-4): 212; Sarkar et al., 2004. Fauna of Bihar (including Jharkhand), Part I, State Fauna Series, 11: 185 .

Common Names: Marbled Narrow-mouthed Frog, Termite Nest Frog, Variegated Balloon Frog, Whitebellied Pug-snout Frog.

Diagnostic Features: Body slender; head small and slightly wider than long, snout rounded to nearly truncated (as seen from above); tympanum hidden; finger tips with triangular dilatations or truncated; toes with rudimentary webbing or none and tips truncated; two metatarsal tubercles, inner distinct; tibio-tarsal articulation reaching shoulder.

Colouration: Dark brown with irregular golden dots and markings.

Snout-Vent Length: 14 mm (Sarkar, 1991); female 31.3 mm (Prasad et al., 2020).

Altitudinal Records: Up to $1,000 \mathrm{~m}$ in India and 3-175 $m$ in Sri Lanka (iucnredlist).

\section{Distribution:}

Bihar: No record.

Jharkhand: Chota Nagpur and West Singhbhum (Chakradharpur) districts.

Rest of India: Andhra Pradesh, Assam, Gujarat, Karnataka (Bengaluru Urban dist.), Kerala (Palakkad and Thrissur dist.), Madhya Pradesh (Panna dist.; Panna Tiger Reserve, Panna and Chhatarpur dists.), Maharashtra (Chandrapur dist.), Odisha, Tamil Nadu (Injambakkam and Villupuram dists.), Telangana and West Bengal (Purba Bardhaman dist.).

Elsewhere: Sri Lanka.

Uncertain: Bangladesh (iucnredlist).

Conservation Status: IUCN Red List- Least Concern.

Remarks: Earlier records of this species from Sri Lanka found to belong to Uperodon rohani Garg et al., 2018 as assessed by Garg et al. (2018).

\section{FAMILY: RANIDAE BATSCH, 1796 \\ Genus: Hylarana Tschudi, 1838 \\ 18. Hylarana erythraea (Schlegal, 1837)}

Hyla erythraea Schlegel, 1837, Abbild. Neuer Unvollst. Amph., 1: 27 (type-locality: Java and Sumatra).

Rana erythraea, Boulenger, 1882, Cat. Batr. Sal. Coll. Brit. Mus., Ed. 2: 65; Boulenger, 1897, Proc. Zool. Soc. London, 1897: 231; Boulenger, 1920. Rec. Indian Mus., 
20: 152-155; Sarkar et al., 1992. Faun. West Bengal, Part 2, State Faun. Series 3; Sarkar and Ray, 2002. Amphibia. In: Fauna of Kabar Lake. Wetland Ecosystem Series, 4: 109.

Common Names: Green-backed Frog, Green Paddy Frog, Golden-lined Frog, Red-eared Frog.

Diagnostic Features: Head longer than broad, interorbital width equal or slightly broader than upper eyelid; tympanum distinct, nearly one eye diameter; fingers slender, with rudimentary webbing, $1^{\text {st }}$ little longer than $2^{\text {nd }}$, tips with distinct discs; subarticular tubercles of fingers and toes well-developed; toes $3 / 4^{\text {th }}$ webbed; two distal phalanges of $4^{\text {th }}$ toe free, $3^{\text {rd }}$ partly or fully webbed, tips with distinct discs; inner metatarsal tubercle oval, outer mostly absent; tibiotarsal articulation reaching between hind edge of eye and snout tip (Sarkar et al., 1992; Sarkar and Ray, 2002, 2006).

Colouration: Dorsum smooth with distinct dorso-lateral glandular folds running between tympanum and vent.

Snout-Vent Length: 37-78 mm (Boulenger, 1920); 3846 mm (Sarkar et al., 1992); 61 mm (Sarkar and Ray, 2006).

Altitudinal Record: Up to 1,200 m (iucnredlist). Distribution:

Bihar: Begusarai (Kabar Lake) district.

Jharkhand: No record.

Rest of India: Andaman and Nicobar Islands, Arunachal Pradesh (Dibang Valley dist.), Assam, Meghalaya, Mizoram, Nagaland, Odisha and West Bengal (Bankura, Howrah, Midnapore Purba Barddhaman and 24 Parganas dists.).

Elsewhere: Bangladesh, Bhutan, Brunei, Cambodia, China, Indonesia, Laos, Malaysia, Myanmar, Nepal, Singapore, Thailand and Viet Nam.

Introduced: Philippines- Negros and Panay (Ferner et al., 2001; Lever, 2003; Frost, 2021).

Conservation Status: IUCN Red List- Least Concern.

Remarks: Ohler and Mallick (2003) refer records of Hylarana erythraea from Bangladesh, India, Myanmar and Nepal to Hylorana tytleri Theobald, 1868 but Dinesh et al. (2009) following Dutta (1997) recognised H. erythraea for India.

Hylarana erythraea differs from $H$. taipehensis (van Denburgh, 1909) occurring in Assam, Meghalaya, Odisha and West Bengal (Dutta, 1997) in having two phalanges of $4^{\text {th }}$ toe free and $3^{\text {rd }}$ phalange of same covered partly or fully with web v/s three free in $H$. taipehensis as noted by Sarkar et al. (1992).

FAMILY: RHACOPHORIDAE HOFFMAN, 1932

Subfamily: Rhacophorinae Hoffman, 1932

Genus: Chirixalus Boulenger, 1893

19. Chirixalus simus Annandale, 1915

Chirixalus simus Annandale, 1915. Rec. Indian Mus., 11: 345-346, fig. 4 (type-locality: Mangaldai, Assam north of Brahmaputra); Sharma and Kumar, 2020. frog leg \# 140. In: Zoo's Print, 35 (9): 18-20, figs.
Chiromentis simus, Deuti et al., 2017. Rec. zool. Surv. India, 117 (3): 236.

Common Names: Annandale's Bush Frog, Annandale's Pygmy Tree Frog, Annandale's Tree Frog. Diagnostic Features: Interorbital space broader than upper eyelid and almost equal to eye diameter; tympanum about $1 / 3^{\text {rd }}-1 / 2^{\text {nd }}$ of eye diameter; fingers with rudimentary webbing, their discs smaller than tympanum, slightly larger than that of those of toes; toes about $2 / 3^{\text {rd }}$ webbed; almost fully webbed (Deuti et al., 2017); subarticular tubercles of fingers and toes well developed; inner metatarsal tubercle small and rather indistinct; tibio-tarsal articulation reaching snout tip (Annadale, 1915).

Colouration: Brownish or pale buff with several indistinct longitudinal dark lines, scattered black specks and small warts.

Snout-Vent Length: $22 \mathrm{~mm}$ (Annandale, 1915); 23.12$27.42 \mathrm{~mm}$ and male 20-24, female 27-30 mm (Deuti et al., 2017).

Altitudinal Record: Below 100 m (iucnredlist).

Distribution:

Bihar: No record.

Jharkhand: East Singhbhum (Dalma Wildlife

Sanctuary, Jamshedpur) district.

Rest of India: Assam, Mizoram and West Bengal (Kodalbasti Forest and Mendabari Forest in Chilapata, Alipurduar dist.).

Elsewhere: Bangladesh.

Uncertain: Bhutan (iucnredlist).

Conservation Status: IUCN Red List- Least Concern.

Genus: Polypedates Tschudi, 1838

20. Polypedates leucomystax (Gravenhorst, 1829)

Hyla leucomystax Gravenhorst, 1829. Delic. Mus. Zool. Vratislav., 1: 26 (type-locality: Java).

Polypedates leucomystax, Chanda, 2002. Handbook Indian Amphibians: 170, 265.

Common Names: Bamboo Tree Frog, Brown Tree Frog, Four-lined Tree Frog, Perching Frog, Striped Tree Frog, White-lipped Tree Frog.

Diagnostic Features: Interorbital width much broader than eye diameter; tympanum distinct, more than $1 / 2$ $3 / 4^{\text {th }}$ eye diameter; fingers free or with rudiment / slightly webbed, $1^{\text {st }}$ finger equal to or slightly longer than $2^{\text {nd }}, 3^{\text {rd }}$ longest; tips of fingers and toes with welldeveloped horse-shoe shaped discs, discs with distinct cutaneous circum-marginal grooves which are comparatively smaller of toes and subarticular tubercles of both small but distinct (Chanda, 1994, $1995 \mathrm{a})$; toes $2 / 3^{\text {rd }}$ webbed, last three digits of $4^{\text {th }}$ free (Chanda, 1994, 1995a) or fully webbed (Chanda, 2002); inner metatarsal tubercle oval and small, outer rudimentary (Chanda, 2002) or absent (Chanda, 1994, 1995a; Sarkar and Ray, 2006) or rudimentary; tibiotarsal articulation reaching anterior margin of eye (Chanda, 1994, 1995) or eye (Chanda, 2002) or between eye and snout tip (Sarkar and Ray, 2006); hind 
limbs when folded at right angles to body, heels strongly overlap (Chanda, 1994).

Colouration: Yellowish, greyish, greenish to reddishbrown with or without dark spots, 3-4 longitudinal bands from snout to vent which may often be faint.

Snout-Vent Length: $55 \mathrm{~mm}$ (Chanda, 1995b); $46 \mathrm{~mm}$ (Chanda, 2002); 53-74 mm (Sarkar and Ray, 2006).

Altitudinal Record: Up to 1,500 m (iucnredlist).

\section{Distribution:}

Bihar: (Chanda, 2002).

Jharkhand: No record.

Rest of India: Andaman and Nicobar Islands, Arunachal Pradesh (Lower Subansiri, Tirap and West Kameng dists.), Assam (Karimganj dist.; Kaziranga National Park, Golaghat, Karbi Anglong and Nagaon dists.), Chhattisgarh (Bastar dist., Indravati Tiger Reserve, Bijapur dist.), Gujarat, Manipur (East and West Imphal dists.), Meghalaya (Cherrapunji, Umiam Lake and Umtynger near Shillong, East Khasi Hills Dist; Ronjeng, East Garo Hills dist.), Nagaland, Sikkim and West Bengal (Jalpaiguri dist.).

Elsewhere: Bangladesh, Borneo, Brunei, Cambodia, China, Indonesia, Laos, Malaysia, Myanmar, Nepal, Papua New Guinea, Philippines, Singapore, Sri Lanka Thailand and Viet Nam.

Introduced: Japan (Sen and Mathew, 2008; iucnredlist). Uncertain: Bhutan (iucnredlist).

Conservation Status: IUCN Red List- Least Concern.

\section{Polypedates maculatus (Gray, 1830)}

Hyla maculata Gray, 1830. Illust. Indian Zool., 1 (3): pl. 82, fig. 1 (type-locality: Bengal [Bangladesh and India]).

Polypedates maculatus, Inger and Dutta, 1968. J. Bombay nat. Hist. Soc., 83 (suppl.): 139; Venkateswarlu and Murthy, 1972. Indian J. Zool, 13 (3): 129; Sarkar, 1991. Rec. zool. Surv. India, 89 (1-4): 216; Sarkar and Ray, 2002. Amphibia. In: Fauna of Kabar Lake. Wetland Ecosystem Series, 4: 111; Sarkar et al., 2004. Fauna of Bihar (including Jharkhand), Part I, State Fauna Series, 11: 191-192; Saniyal et al., 2014. Rec. zool. Surv. India, Occ. Paper No. 361: 20; Kumar, 2019. frog leg \# 138. In: Zoo's Print, 34 (8):13, 16, fig.; Pankaj, 2020. International Research Journal of Modernization in Engineering Technology and Science, 2 (1): 79, 80, 81; Sharma and Kumar, 2020. frog leg \# 140. In: Zoo's Print, 35 (9): 18, 20; Pankaj and Nath, 2021a. Reptiles and Amphibians, 28 (1): 107, fig. 2H; Pankaj and Nath, 2021b. MOJ Biology and Medicine, 6 (2): 61 .

Common Names: Common Tree Frog, Indian Tree Frog, Spotted Tree Frog, Spotted Whipping Tree Frog.

Diagnostic Features: Interorbital width broader than upper eyelid; tympanum distinct, nearly $1 / 3^{\text {rd }}$ or about $3 / 4^{\text {th }}$ of eye diameter; fingers with rudimentary webbing, $1^{\text {st }}$ equals $2^{\text {nd }}$ (Sarkar and Ray, 2006); tips of fingers and toes with distinct horse-shoe-shaped discs (Chanda, 2002; Sarkar and Ray, 2002, 2006; Sarkar et al., 2004); webbing not extending up to discs, two phalanges of $1^{\text {st }}, 2^{\text {nd }}$ and $3^{\text {rd }}$ toes free except for a minor fringe, three phalanges of $4^{\text {th }}$ toe without webbing, only $5^{\text {th }}$ toe webbed up to base of disc (Ray, 1999); subarticular tubercles of fingers and toes distinct; toes nearly $3 / 4^{\text {th }}$ webbed, two distal phalanges of $4^{\text {th }}$ toe free; inner metatarsal tubercle oval and distinct, outer absent (Ray, 1999; Sarkar et al., 2004); tibio-tarsal articulation reaching snout tip (Ray, 1999) or between hind edge of eye and snout tip (Sarkar and Ray, 2002, 2006; Sarkar et al., 2004).

Colouration: Golden brownish with dark brown spots. Snout-Vent Length: 43-68 mm (Sanyal et al., 1991); 11-62 mm (Sarkar, 1991); 42-53 mm (Ray and Tilak, 1995); 35-65 mm (Chanda, 2002); 15-73 mm (Sarkar et al., 2004); 38-43 mm (Sarkar and Ray, 2006); 35-85 mm (Ingle, 2011); males 34-57, females 44-89 mm (Husain, 2015); 35-65 mm (Ingle, 2020); male 43.346.9 , female 60.3 and individuals (sex not known) 38.8$51.9 \mathrm{~mm}$ (Prasad et al., 2020).

Altitudinal Records: Up to $700 \mathrm{~m}$ (Ray, 1999); 1,500 m (Husain, 2015; iucnredlist); below 3,000 m (Frost, 2021).

\section{Distribution:}

New Record: Erki, Jehanabad (Bihar).

Bihar: Aurangabad (Raja Jagannath Dam Deo), Begusarai (Kabar Lake), Bhagalpur, Gaya, Nalanda, and Rohtas districts.

Jharkhand: Deoghar (Kali Mandir area), Dhanbad, Dumka (Asanbani), East Singhbhum (Dalma Wildlife Sanctuary, Jamshedpur), Palamau, Ranchi, Seraikela Kharsawan (Chandil) and West Singhbhum (Tholkabad) districts.

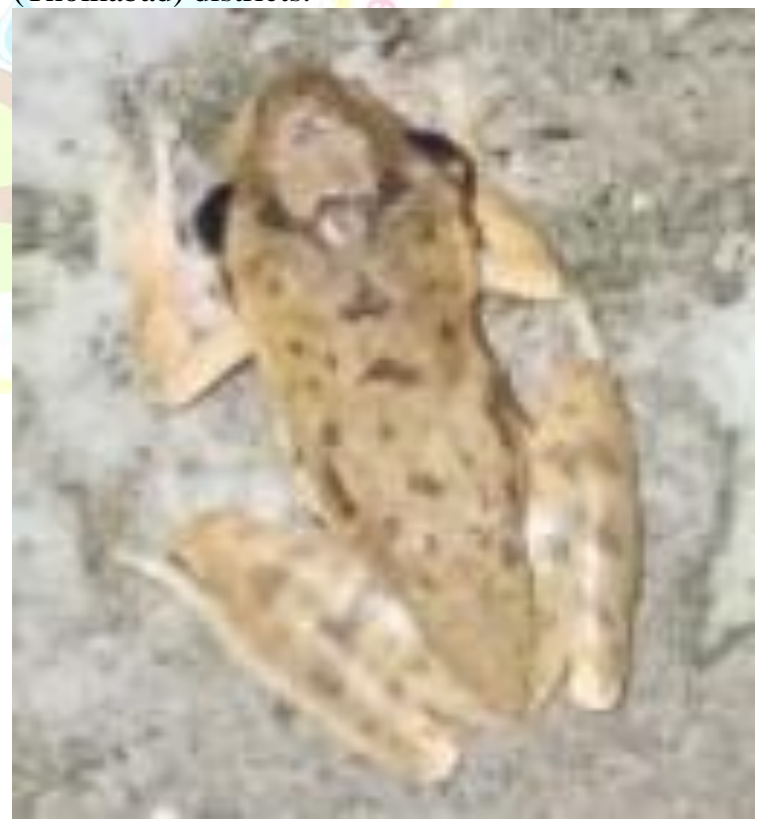

Figure 5a. Polypedates maculatus, Common Tree Frog (from Jehanabd, Bihar, by $2^{\text {nd }}$ author)

Rest of India: Arunachal Pradesh (East Siang, Tirap and West Kameng dists.), Chhattisgarh (Bastar, Bijapur, Bilaspur, Dantewada, Dhamtari, Jashpur, Kanker, 
Kondagaon, Narainpur and Sukma dists.; Indravati Tiger Reserve, Bijapur dist.; Kanger Valley National Park, Bastar dist.), Goa (North Goa and South Goa dists.), Gujarat (Dang dist.), Karnataka (Bengaluru Urban, Bengaluru Rural, Shivamogga and Uttar Kannada dists.), Kerala (Kollam and Thrissur dists.), Madhya Pradesh (Dinori, Hoshangabad, Jabalpur, Seoni, Sheopur and Ujjain dists.; Panna Tiger Reserve, Panna and Chhatarpur dists.; Pench Tiger Reserve, Seoni dist.), Maharashtra (Chandrapur, Malvan, Pune, Raigad, Ratnagiri, Sangli, Sindhudurg and Thane dists.), Meghalaya, Odisha (Balighat Falls , Koraput dist.), Rajasthan (Dungarpur dist.), Sikkim, Tamil Nadu (Chennai dist.), Telangana (Medak dist.), Uttarakhand (Almora, Dehra Dun, Pauri and Uttarkashi dists.; Gobind Pashu Vihar, Uttarkashi dist.; Rajaji TigerReserve, Dehra Dun, Haridwar and Pauri dists.), Uttar Pradesh and West Bengal (Bankura, Darjeeling, Kolkata, Midnapore, Purba Bardhaman, Purulia and South 24 Parganas dists.).

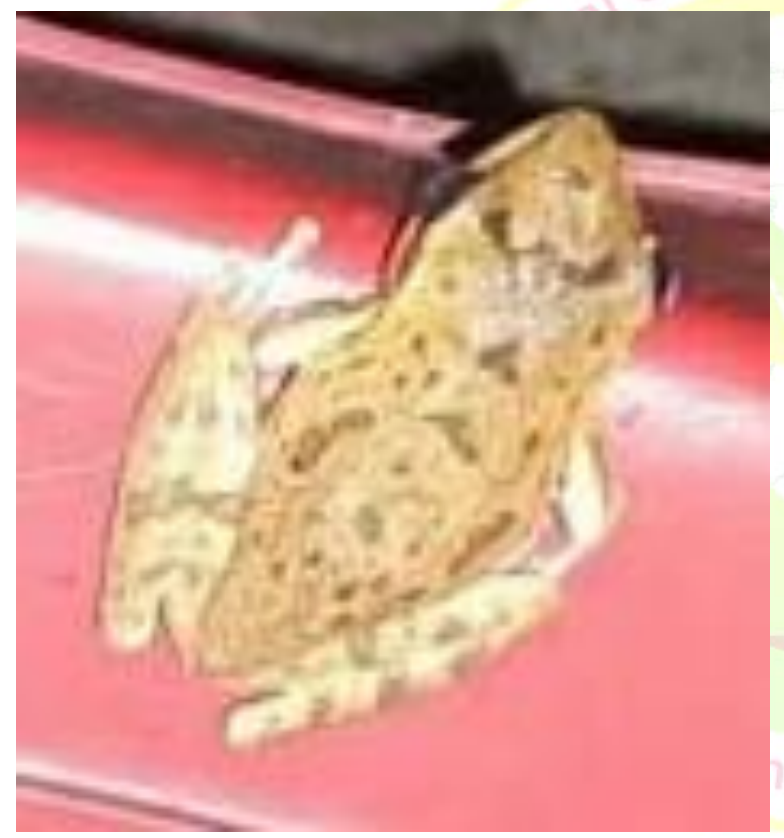

Figure 5b. Polypedates maculatus, Common Tree Frog (from Jehanabad, Bihar, by $2^{\text {nd }}$ author)

Note: Inger and Dutta (1986), Senand Mathew (2008) and Frost (2021) recorded from all over India except Haryana, Rajasthan and Punjab. Frost (2021) also opined same for north-eastern states of India.

Elsewhere: Bangladesh, Bhutan, Nepal, Sri Lanka.

Uncertain: China and Myanmar (Husain, 2015; iucnredlist).

Conservation Status: IUCN Red List- Least Concern.

\section{Polypedates taeniatus (Boulenger, 1906)}

Rhacophorus taeniatus Boulenger, 1906. J. Asiat. Soc. Bengal, Ser. 2, 2: 385 (type-locality: Purneah, Bengal [Bihar]).
Polypedates taeniatus, Deuti et al., 2018. Rec. zool. Surv. India, 118 (3): 298-306.

Common Names: Bengal Whipping Tree Frog, Ribboned Tree Frog, Terai Reed Frog, Terai Tree Frog.

Diagnostic Features: Slender and small-sized body; head slightly longer, dorsally flattened and triangular; eyes large; fingers and toes with rudimentary webbing, tips dilated into discs; inner metatarsal tubercle elongated.

Colouration: Brown to golden yellow with six longitudinal dark brown stripes; tympanum dark tan; limbs uniform without any markings; ventrum whitish; thighs pinkish.

Snout-Vent Length: 41.37-42.46 mm (Deuti et al., 2018).

Altitudinal Records: Below 500 m (Deuti et al., 2018; iucnredlist); $208 \mathrm{~m}$ at Bidurkuti, Bijnor, Uttar Pradesh (Boruah and Das, 2020).

\section{Distribution:}

Bihar: Purnia district.

Jharkhand: No record.

Rest of India: Assam (Orang National Park, Sonitpur and Udalguri dists.), Manipur, Meghalaya, Mizoram, Nagaland, Uttar Pradesh (Bijnor, Meerut and Moradabad dists.; Dudhwa National Park, Lakhimpur Kheri dist.; Katarniaghat Wildlife Sanctuary, Bahraich dist.) and West Bengal (Behala Aerodroma, Kolkata).

Elsewhere: Bangladesh and Nepal.

Uncertain: Bhutan (iucnredlist).

Wangyal et al. (2020) tentatively identified a photograph from Bhutan (Samdrup Jongkhar district).

Conservation Status: IUCN Red List- Least Concern.

Remarks: Ray (1991) made studies on its distribution, biology and osteology. Deuti et al. (2018) studied its breeding call and post-embryonic development stages in detail. Boruah and Das (2020) recorded it from Bijnor district (Uttar Pradesh) and studied predation on its eggs by Flesh-fly larvae (Diptera).

\section{OBSERVATIONS}

Inter-orbital width, tympanum size, finger and toe tips shape and their webbing, subarticular tubercles, metatarsal tubercles, extent of tibio-tarsal articulation and colouration found varied as per various authors who dealt with material from different biotopes.

\section{POTENTIAL BIOLOGICAL CONTROLLERS}

Anurans (frogs and toads), being predators on a variety of insects (Coleoptera, Diptera, Lepidoptera, Diptera, Hemiptera and Orthoptera) and other arthropods, are of great help in controlling them, especially in paddy fields and other water filled sites of agricultural importance. The insects (including pests) are considered harmful to paddy crops and other vegetation, inflicting great loss in rice production and other seasonal crops. Studies have shown that a good population of frogs can keep paddy fields free of various insects. Besides this, Khatiwada et al. (2016) also found them consuming a large number of other insects which are known as important vectors of 
zoonotic diseases and opined to encourage farmers and other planners to consider anurans also as one of the important biological controllers for the management of insects. Propper et al. (2020) also provided role of amphibians in rice farming. The commonly used agrochemicals / pesticides (insecticides, fungicides etc.) in fields may also kill frogs and hence should be avoided as far as possible, especially directly on water pockets as they breed there and their eggs / tadpoles may be there. Arboreal or Tree frogs (Chirixalus simus and Polypedates spp.) can help to some extent in controlling wood borers. They are also helpful in controlling mosquitoes as their tadpoles mainly subsist on their larval stage. Earlier, Raghavendra et al. (2008) and Naz et al. (2019) dealt with biological control of mosquitoes through anuran tadpoles.

\section{CONCLUSION}

A total of 22 species belonging to 12 genera, 5 families under order Anura have been recorded from both Bihar and Jharkhand. Presently, Bihar is richer in having 19 species under 11 genera and 5 families than Jharkhand with 15 spp. under 10 genera and 4 families. Four new records (Firouzophrynus stomaticus, Haplobatrachus tigerinus, Uperodon taprobanicus and Polypedates maculatus) from Jehanabd (Bihar) have been added. However, further extensive surveys may reveal the same species with more records from both the states. As regards generic diversity, Uperodon is the richest, with 4 spp. covering maximum $18.18 \%$, Sphaerotheca and Polypedates with 3 spp. each $13.64 \%$, Hoplobatrachus, Kaloula and Microhyla with 2 spp. each $9.09 \%$ and Chirixalus, Duttaphrynus, Firouzophrys, Euphylyctis, Hylorana and Occidozyga with 1 sp. each $4.55 \%$. Family wise percentage of species: Microhylidae $36.36 \%$ most abundant, with 8 species, Dicroglossidae $31.81 \%$ with 7, Rhacophoridae $18.18 \%$ with 4 , Bufonidae $9.09 \%$ with 2 and Ranidae $4.55 \%$ with single, the least. Fejervarya limnocharis is actually known from Andaman and Nicobar Islands (India), Cambodia, Indonesia, Laos, Malaysia, Myanmar, Thailand and Vietnam as per molecular and morphological studies. The populations (as $F$. limnocharis) from India (main land), Bangladesh, China, Indonesia, Japan, Nepal, Philippines, Sri Lanka and Taiwan, associated with this name, are currently considered unnamed species and hence not included here.

Bufo bufo and Litoria alboguttata (= Ranoidea alboguttata), the European and Australian forms respectively, reported from Gautam Buddha Wildlife Sanctuary (Bihar and Jharkhand) appear misidentifications for Duttaphrynus melanostictus and Kaloula assamensis or K. pulchra, the species found in Bihar and hence not included here.

Frogs, being predators on a variety of insect and other arthropods, including pests, play an important role in controlling them, especially in paddy fields and other water filled sites of agricultural importance. They are also helpful in controlling mosquitoes. Tree Frogs can be helpful in wood borer control.

\section{ACKNOWLEDGEMENTS}

The authors are thankful to Dr H. J. Husain, Head Biodiversity, Corporate Sustainability Centre of Excellence for Biodiversity Management, Corporate Sustainability, Tata Steel Ltd., Commercial Centre, Jamshedpur, Jharkhand, India, Mr. C. Radhakrishnan, (former Scientist-F, Zoological Survey of India), 33/ 5267-D, Golf Links Road, Chevayur, Kozhikode, Kerala and Dr. S. S. Talmale, Scientist-B, Western Regional Centre, ZSI, Rawet Road, Pune, Maharashtra, India for their help.

\section{REFERENCES}

Annandale, N. 1915. Herpetological notes and descriptions. Rec. Indian Mus., 11: 341-457.

Annandale, N. and Rao, C. R. N. 1918. The tadpoles of families Ranidae and Bufonidae found in plains of India. Rec. Indian Mus., 15 (1): 25-41.

Bhaduri, J. L. 1944. A note on Rana crassa Jerdon, with extension of range. J. Bombay nat. Hist. Soc., 44 (3): 481-484.

Bhaduri, J.L. and Kirpalani, M.R. 1954. Note on the frog Rana breviceps Schneider. J. Bombay nat. Hist. Soc., 52 (4): 620-623.

Biju, S.D. 2001. A synopsis of the frog fauna of the Western Ghats, India: 1-24. Occasional Publication, Indian Society for Conservation Biology, Tropical Botanic Garden and Research Institute, Thiruvananthapuram, Kerala (India).

Boruah, B. and Das, A. 2020. Predation on an egg mass of the Terai Treefrog, Polypedates taeniatus (Anura: Rhacophoridae), by Flesh Fly larvae (Diptera: Sarcophagidae). IRCF Reptiles and Amphibians, 27 (2): 228-230.

Boulenger, G. A. 1920. A monograph of the South Asian, Papuan, Melanesian and Australian frogs of the genus Rana. Rec. Indian Mus., 20: 1-126.

Boulenger, G.A. 1890. The Fauna of British India, including Ceylon and Burma. Reptilia and Batrachia: xvii + 541. Taylor and Francis, London.

Chanda, S. K. 1994. Anuran (Amphibia) Fauna of Northeast India. Mem. zool. Surv. India, 18 (2): $1-143$.

Chanda, S. K. 1995a. Anuran (Amphibia). In: Fauna of Meghalaya, Part-1. State Fauna Series, 4: 455482. Zoological Survey of India Publication.

Chanda, S. K., 1995b. Amphibia: 71-75. In: Fauna of Indravati Tiger Reserve. Fauna of Conservation Areas, No. 6. ZSI Publication.

Chanda. S. K. 2002. Hand Book Indian Amphibians: 1273, 39 pls. Zoological Survey of India Publication.

Chandra, K. and Gajbe, P. U. 2005. An inventory of herpetofauna of Madhya Pradesh and 
Chhattisgarh. Zoo's Print Journal, 20 (3): 18121819.

Christy, M. T., Clark, C. S., Gee, D. E., Vice, D., Vice, D. S., Warner, M. P., Tyrrell, C. L., Rodda, G. H. and Savidge, J. 2007. Recent records of alien anurans on the Pacific island of Guam. Pacific Science, 61: 469-483.

Daniel, J. C., 2002. The Book of Indian Reptiles and Amphibians: viii +238 pp. Bombay Natural History Society and Oxford University Press, Mumbai.

Deuti, K., Murthy, B. H. C. K. and Sethy, P. G. S. 2018. A new distribution record of Polypedates taeniatus (Amphibia: Anura: Rhacophoridae) with notes on its breeding call and postembryonic development stages. Rec. zool. Surv. India, 118 (3): 298-306.

Deuti, K., Ray, S., Bag, P. and Dey, S.K. 2017. Amphibians of the Duars area of northern West Bengal. Rec. zool. Surv. India, 117 (3): 221-224.

Deuti, K., Sethy, P. G. S. and Ray, S. 2014. Amphibians of the Eastern Ghats. Rec. zool. Surv. India, 114 (Part-1): 119-144.

Deuti, K., Sethy, P.G.S., Ray, S. and Chattopadhyay, M. 2012. First record of Kaloula assamensis Das et al. (2004) (Microhylidae) from Bihar State, India, with notes on its distribution. J. Bombay nat. Hist. Soc., 109 (3): 208-209.

Dinesh, K.P., Radhakrishnan, C., Gururaja, K.V. and Bhatta, G.K. 2009. An annotated checklist of amphibians of India with some insight into the pattern of species discoveries, distribution and endemism. Rec. zool. Surv. India, Occ. Pap. No. 302: 1-153.

Dinesh, K.P., Radhakrishnan, C., Channakeshavamurthy, B.H., Deepak, P. and Kulkarni, N.U. 2020. A Checklist of Amphibians of India with IUCN Conservation Status. Version 3.0. updated till April 2020 (available at http://zsi.gov.in).

Dubois, A. 1983. Note preliminaire sur le groupe de Rana (Tomopterna) breviceps Schneider,1799 (Amphibiens, Anoures), avec diagnose d'une sous-expèce nouvelle de Ceylan. Alytes, 2: 163170.

Dubois, A. and Ohler, A. 1999. Asian and oriental toads of the Bufo melanostictus, Bufo scaber and Bufo stejnegeri groups (Anura, Amphibia): a list of available and valid names and redescription of some name-bearing types. J. South Asian Nat. Hist., 4 (2): 133-180.

Dutta, S. K. 1997. Amphibians of India and Sri Lanka (checklist and bibliography): 1-342. Odyssey Publishing House, Bhubaneshwar, India

Dutta, S. K. 2015. Anuran fauna of Bastar Division of Chhattisgarh state, India. Ambient Science, 2 (2): 31-38, figs.

Ferner, J. W., Brown, R. M., Sison, R. V. and Kennedy, R.S. 2001. The amphibians and reptiles of Panay
Island, Philippines. Asiatic Herpetological Research, 9: 34-70.

Frost, D. R. 2021. Amphibian Species of the World: An Online Reference. Version 6.1 (Date of access). https://amphibiansoftheworld.amnh.org/index.ph p. American Museum of Natural History, New York, USA. doi.org/10.5531/db.vz.0001 .

Garg, S., Senevirathne, G., Wijayathilaka, N., Phuge, S., Deuti, K., Manamendra, K., Meegaskumbura, N. and Biju, S. D. 2018. An integrative taxonomic review of the South Asian microhylid genus Uperodon. Zootaxa, 4384: 1-88.

Garg, S., Suyesh, R., Das, A., Jiang, J.P., Wijayathilaka, N., Amarasinghe, A.A.T., Alhadi, F., Vineeth, K. K., Aravind, N.A., Senevirathne, G., Meegaskumbura, M. and S.D. Biju, S.D. $2019 . \quad$ Systematic revision of Microhyla (Microhylidae) frogs of South Asia: a molecular, morphological, and acoustic assessment. Vertebrate Zoology, 69: 171.

Husain, 2015. Amphibians of Doon Valley (Dehra Dun, Uttarakhand) with their systematic distribution, ecology, conservation status and threats In: Aquatic Ecosystem: Biodiversity, Ecology and Conservation: 217-229.

Husain, A. and Husain, H. J. 2020. New record of Duttaphrynus melanostictus (Schneider, 1799) (Anura: Bufonidae) from Jamshedpur, Jharkhand with systematic account and conservation status. International Journal of Theoretical and Applied Sciences, 12 (2): 23-26, figs. 1-5.

Husain, A., Dubey, A. K. and Husain, H. J. 2020. New record of Duttaphrynus stomaticus (Lutken, 1864) (Anura: Bufonidae) from Aligarh, U.P., with systematic account, conservation status and differences from D. melanostictus (Schneider, 1799). International Journal of Global Science Research, 7 (2): 1432-1437, figs. 1, 2.

Hussain et al. 2017. An assessment report on biodiversity value of Gautam Buddha Wildlife Sanctuary, Bihar and Jharkhand. TR No. 2017/01 6.78 pp. Dehradun, India.

Inger, R. F. and Dutta, S. K. 1986. An overview of the amphibian fauna of India. J. Bombay nat. Hist. Soc., 83 (suppl.): 135-146.

Ingle, M., 2011. Herpetofauna of Naglok region, Jashpur district, Chhattisgarh. Rec. zool. Surv. India, 111 (Part-4): 79-96.

Ingle, M., 2020. Herpetofauna of the Amarkantak Plateau in Central India. Reptiles and Amphibians, 27 (3): 397-410.

Khajeh, A., Mohammadi, Z., Ghorbani, F., Meshkani, J., Rastegar-Pouyani, E. and Torkamanzehi, A. 2014. New insights into the taxonomy of the skittering frog Euphlyctis cyanophlyctis complex (Schneider, 1799) (Amphibia: Dicroglossidae) based on mitochondrial $16 \mathrm{~S}$ 
rRNA gene sequences in southern Asia. Acta Herpetologica, 9: 159-166.

Kumar, R. 2019. Amphibian diversity of Rajgir Wildlife Sanctuary, Bihar, India. frog leg \# 138. In: Zoo's Print, 34 (8): 12-17, figs.

Lever, C. 2003. Naturalized Reptiles and Amphibians of the World. New York: Oxford University Press.

Manamendra-Arachchi, K. and Pethiyagoda, R. 1998. A synopsis of the Sri Lankan Bufonidae (Amphibia: Anura) with description of new species. J. South Asian Nat. Hist., 3: 245.

Meegaskumbura, M., Biju, S. D., Karunarathna, N., Senevirathne, G., Garg, S. and Wijayathilaka, N. 2016. A new species of Microhyla (Anura: Microhylidae) from Sri Lanka: an integrative taxonomic approach. Zootaxa. 4066 (3): 331342.

Naz, S., Huma Akbar, H., Habib, S. S., Faiq, M., Rais, M., Malik, M. A. and Shafiq, A. 2019. Role of anuran tadpoles as biological control for dengue larvae. International Journal of Mosquito Research, 6 (3): 22-26.

Ohler, A. and Mallick, P.K. 2003. Rana (Hylarana) sensu Dubois (1992) in India and the identity of Hylorana tytleri Theobald, 1868. Hamadryad, 27: 62-70.

Pankaj, N. and Nath, B. 2021a. Anurans of Gaya district, Bihar, India. Reptiles and Amphibians, 28 (1): 106-109.

Pankaj, N. and Nath, B. 2021b. Habitat preferences and species diversity of anuran amphibians of Gaya, India. MOJ Biology and Medicine, 6 (2): 58-63.

Pankaj, N., 2020. Evaluation of amphibian fauna of the different forest and grassland habitats in and around the area of Raja Jagannath Bandh Deo India. International Research Journal of Modernization in Engineering Technology and Science, 2 (1): 75-84, figs. 1-4.

Parker, H. W. 1934. A monograph of the frogs of family Microhylidae: viii + 208, 67 figs. London (Trustees of British Museum).

Prasad, V. K., Dinesh, K. P., Das, A., Swamy, P., Shinde, A. D. and J. B. Vishnu, J. B. 2019. A new species of Sphaerotheca Gunther, 1859 (Amphibia: Anura: Dicroglossidae) from the agro ecosystems of Chota Nagpur Plateau, India. Rec. zool. Surv. India, 119 (3): 197-210.

Prasad, V. K., Gautam, K. B., Gupta, S. K., Murthy, R. S., Ramesh, K. Shinde, A. D. and Das, A. 2020. Identification of anuran species diversity of the Panna Tiger Reserve, Central India, using an integrated approach. Zootaxa, 4851 (3): 450-476.

Propper, C. R., Hardy, L. J., Howard, B. D., Flor, R. J. B and Singleton, G. R. 2020. Role of farmer knowledge in agroecosystem science: rice farming and amphibians in the Philippines. Human-Wildlife Interactions, 14 (2):273-286.

Raghavendra, K. R., Sharma, P. and Dash, A. P. 2008. Biological control of mosquito populations through frogs: Opportunities and constraints. Indian J. Med. Res., 128: 22-25.

Rao, N. V. A. and Shukla, R. K. 2014. Occurrence of cannibalism in adult Euphlyctis cyanophlyctis (Anura; Ranidae): Is it induced or opportunistic? International Research Journal of Natural and Applied Sciences, 1 (6): 186-190, figs. 1a, b.

Ray, P. 1991. On natural distribution of the rare tree frog Rhacophorus taeniatus Boulenger, 1906 (Anura: Rhacophoridae), with notes on its biology and osteology. J. Bombay nat. Hist. Soc., 88 (3): 276-380.

Ray, P. 1999. Systematic studies on the amphibian fauna of the district Dehradun, Uttar Pradesh, India. Mem. Zool. Surv. India, 18 (3): 1-102.

Ray, P. and Tilak, R., 1995. Amphibia: 55-75, figs. In: Fauna of Rajaji National Park. Fauna of Conservation Areas, 5. ZSI Publication.

Ray, S. 1998. Amphibia: 43-50. In: Fauna of Valmiki Tiger Reserve. Fauna of Conservation Area, No. 10. Zoological Survey of India Publication.

Sanyal, D. P., Chandra, P. K. and Ray, S., 1991. Notes on Amphibian collection from Bastar district, Madhya Pradesh, India. Rec. zool. Surv. India, 88 (1): 45-48.

Sarkar, A. K. 1991. The Amphibians of Chota Nagpur (Bihar), India. Rec. zool. Surv. India, 89 (Part 14): 209-217.

Sarkar, A. K. and Ray, S. 2002. Amphibia. In: Fauna of Kabar Lake. Wetland Ecosystem Series, 4: 107112. Zoological Survey of India Publication.

Sarkar, A. K. and Ray, S. 2006. Amphibia. Fauna of Arunachal Pradesh (Part-1), State Fauna Series, 13: 285-316. Zoological Survey of India Pub.

Sarkar, A. K., Biswas, M. L. and S. Ray, S. 1992. Amphibia. In: Fauna of West Bengal, Part-2. State Fauna Series, 3: 67-100. ZSI Publication.

Sarkar, A. K., Das, S. and Ray, S. 2004. Amphibia: 181-193. In: Fauna of Bihar (including Jharkhand), Part I, State Fauna Series, 11. Zoological Survey of India Publication.

Sen, N. and Mathew, R. 2008. Bibliographical notes on the amphibians of north east India. Rec. zool. Surv. India, Occ. Paper No., 291: 1-58.

Shah, K. B. and Tiwari, S. 2004. Herpetofauna of Nepal: 37. A Conservation Companion. Kathmandu, Nepal: IUCN Nepal- The World Conservation Union.

Sharma, G. and Kumar, R. 2020. A new distributional record of Annandale's Bush Frog from Dalma Wildlife Sanctuary, Jharkhand. frog leg \# 140. In: Zoo's Print, 35 (9): 18-20, figs.

Siler, C. D., Welton, L. J., Siler, J. M., Brown, J., Bucol, A., Diesmos, A. C., and R. M. Brown, R. M. 2011. Amphibians and reptiles, Luzon Island, Aurora Province and Aurora Memorial National Park, northern Philippines: New island distribution records. Check List. A Journal of Species Lists and Distribution, 7: 182-195. 
Srinivasulu, C., Srinivasulu, B. and Rao, C. A. N. 2006. Amphibian fauna of Nagarjunasagar Srisailam Tiger Reserve, Andhra Pradesh. Rec. zool. Surv. India, Occ. Paper No. 245: 1-57 (4 pp Plates).

Subba, B., Aravind, N. A. and Ravikanth, G. 2016. Amphibians of the Sikkim Himalaya, India: an annotated checklist. Check List. The Journal of Biodiversity Data, 13(1: 2033): 1-14.

Taylor, E. H., 1962. The amphibian fauna of Thailand. University of Kansas Science Bulletin, 43: 265599.

Venkateswarlu, T. and Murthy, T. S. N., 1972. Fauna of Bihar state (India), 2-Amphibia. Indian J. Zool., 13 (3): 129-130.

Verma, G.B.S.N.P. 2019. New frog species from unexplored Jharkhand and Bihar bring cheer. Mongabay-India.

(http://India.mongabay.com/2019/09/newfrogspecies-from-unexplored-jharkhand-and-biharbrings-cheer/. Accessed on 29 December 2020).
Wangyal, J. T., Bower, D. S., Sherub, Tshewang, S., Wangdi, D., Rinchen, K., Phuntsho, S., Tashi, C., Koirala, B. K., Bhandari, G. S., Phuntsho, Y., Koirala, T. P., Ghalley, B. B., C, L., Tenzin, J., Powrel, R. B., Tshewang, R., Raika, O. N., Jamtsho, S., Kinley, Gyeltshen, Tashi, S., Nidup, D., Wangdi, N., Phuentsho, Norbu, L., Wangdi, K., Wangchuk, T., Tobgay, P., Dorji, T. and Das, I. 2020. New herpetofaunal records from the Kingdom of Bhutan obtained through citizen science. Herpetological Review, 51: 790-798.

Zhao, E.-m., and Adler, K. A. 1993. Herpetology of China. Oxford, Ohio. Contributions to Herpetology, 10. Society for the Study of Amphibians and Reptiles.

https://amphibiaweb.org https://www.ecologyasia.com https://en.wikipedia.org https://www.inaturalist.org https://www.iucnredlist.org

Citation: Husain, A. and Hasan, W. 2021. Review and Updating: Anurans (Amphibia) of Bihar and Jharkhand, India, with some new records from Jehanabad (Bihar) and their role as potential biological controllers. International Journal of Agricultural and Applied Sciences, 2(1): 1-23. https://doi.org/10.52804/ijaas2021.211

Copyright: (C) Husain and Hasan 2021. Creative Commons Attribution 4.0 International License. IJAAS allows unrestricted use, reproduction, and distribution of this article in any medium by providing adequate credit to the author(s) and the source of publication. 